A C T A CHE M I C A S C A N D I N A I C A 26 (1972) 3819-3833

\title{
Crystal Structure of L-Tyrosine
}

\author{
ARVID MOSTAD, HANS MARTIN NISSEN and \\ CHRISTIA N R M M I N G \\ Department of Chemistry, University of Oslo, Oslo 3, Norway
}

\begin{abstract}
The crystal structure of L-tyrosine has been determined by $\mathrm{X}$-ray methods using 2259 unique reflections collected by counter diffractometer techniques. The unit cell is orthorhombic, space group $P 22_{1} 2_{1}$, with dimensions $a=6.91_{3} \AA, b=21.11_{6} \AA, c=5.82_{9} \AA$, and contains four molecules. The structure was solved by direct methods and refined to a conventional $R$ factor of 0.049 by least-squares methods. Estimated standard deviations in bond lengths not involving hydrogen atoms are $0.002 \AA$ and in angles $0.1-0.2^{\circ}$. The conformation about the $\mathrm{C} 7-\mathrm{C} 8$ bond is staggered with the $\mathrm{C} 8$ hydrogen atom anti relative to the phenyl ring. The crystal structure is stabilized through a three-dimensional network of hydrogen bonds.
\end{abstract}

L-Tyrosine plays an important role in the living organism, not only as a building unit in proteins but also by taking part in the biosynthesis of

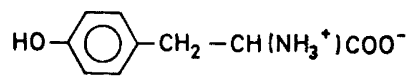

hormones, neurotransmitters, and pigments. During the last 20 years several papers on the structure of salts and derivatives of this compound have been published as contributions to the physical characterization of the molecule. Crystal structure data on L-tyrosine itself have been lacking, however, presumably because of the difficulty of obtaining crystals suitable for X-ray examination. In the course of the structure investigation of several biologically important amino acids a method of growing single crystals of L-tyrosine has been found. The crystallization procedure as well as a brief description of the result of the X-ray crystal structure analysis have been published elsewhere. ${ }^{1,2}$ In the present report we are presenting a more detailed account of the structure determination and a discussion of the crystal and molecular structure of this amino acid.

\section{EXPERIMENTAL}

Commercially obtained $\mathrm{I}_{\text {-tyrosine }}$ was recrystallized according to the method given in Ref. 1. A single crystal of dimensions $0.3 \times 0.4 \times 0.7 \mathrm{~mm}^{3}$ was used in all X-ray experiments; it was mounted with the direction of the largest dimension, the crystallographic $c$ axis, along the goniometer axis.

Acta Chem, Scand. 26 (1972) No. 10 
Oscillation and Weissenberg photographs indicated orthorhombic symmetry. The extinctions uniquely determined the space group to be $P 2_{1} 2_{1} 2_{1}$ in agreement with the result obtained by Boggs and Donohue. ${ }^{3}$ Unit cell dimensions were determined from diffractometer measurements on five general reflections and their Laue-symmetric equivalents. A manual Picker diffractometer was applied using CuK $\beta$ radiation $(\lambda=$ $1.3922 \AA$ ) and a take-off angle of $0.5^{\circ}$. The computer program used in the least-squares calculations of cell parameters, as well as the programs employed during the subsequent calculations are parts of an assembly of programs for a CD-3300 computer and described in Ref. 4.

Three-dimensional intensity data were recorded with the use of an automatic Picker four-angle diffractometer using graphite crystal monochromated MoK radiation. The take-off angle was $4^{\circ}$ and the temperature was kept constant to within $1^{\circ}$ at $18^{\circ} \mathrm{C}$. Diffraction data for 2948 reflections with $2 \theta<80^{\circ}$ were measured using the $\omega-2 \theta$ scanning mode with a $2 \theta$ scan speed of $2^{\circ}$ min $^{-1}$ through the scan range from $0.55^{\circ}$ below $2 \theta\left(\alpha_{1}\right)$ to $0.65^{\circ}$ above $2 \theta\left(\alpha_{2}\right)$. Background counts were taken for 20 sec at each of the scan range limits. Three standard reflections measured after every 100 reflections showed no significant fluctuation during the experiment. The 2259 reflections greater than $2 \sigma(I)$ were considered to be observed; the remaining 689 reflections were excluded from the structure refinement procedure. Lorentz and polarization corrections were applied to the intensity data.

Atomic form factors used were those of Hanson et al..$^{*}$ for oxygen, nitrogen, and carbon, and of Stewart et al..$^{6}$ for hydrogen.

\section{CRYSTAL DATA}

L-4-Hydroxyphenylalanine (L-tyrosine) $\mathrm{C}_{9} \mathrm{H}_{11} \mathrm{O}_{3} \mathrm{~N}$, orthorhombic. $a=$ $6.913(0.005) \AA ; b=21.116(0.003) \AA ; c=5.829(0.004) \AA$. Figures in parentheses are estimated standard deviations. $V=850.9 \AA^{3} ; M=181.19 ; F^{\prime}(000)=384$; $\left(Z=4\right.$. $D_{\text {obs }}$ (flotation) $=1.41 \mathrm{~g} \mathrm{~cm}^{-3}, D_{\text {calc }}=1.414 \mathrm{~g} \mathrm{~cm}^{-3}$. Absent reflections: $(h 00)$ for $h$ odd, $(0 k 0)$ for $k$ odd, (00l) for $l$ odd; space group $P 2_{1} 2_{1} 2_{1}$.

\section{DETERMINATION OF THE STRUCTURE}

The phases of 400 normalized structure amplitudes were obtained by the application of the symbolic addition procedure and the tangent formula..$^{7,8}$ Three-dimensional $F$-maps were calculated with eight sets of phases for the 400 structure factors and in one of the maps the 13 largest peaks were consistent with the non-hydrogen atoms of a reasonable model for the $\mathrm{L}$-tyrosine molecule.

Preliminary refinements were carried out by the minimum residual method ${ }^{9}$ followed by three cycles of least squares refinement. At this stage stereochemically reasonable hydrogen atomic positions were postulated and anisotropic thermal parameters for non-hydrogen atoms introduced. Several full-matrix least-squares refinement cycles were calculated resulting in a conventional $R$ value of 0.049 and a weighted $R$ of 0.046 . The overdetermination ratio was $13.9,162$ parameters being refined on the basis of 2259 reflections with $\sin \theta / \lambda<0.9 \AA^{-1}$.

A comparison of observed and calculated structure factors is given in Table 1; the final parameters for non-hydrogen atoms are listed in Table 2, and for hydrogen atoms in Table 3.

Magnitudes and directions of the principal axes of the ellipsoids of vibration are given in Table 4. An analysis of the thermal parameters showed that the phenol and alanine parts may be regarded separately as rigid bodies 
Table 1. Observed and calculated structure factors. The columns are $h, k, l, 10 F_{\mathrm{o}}$ and $10 F_{c}$.

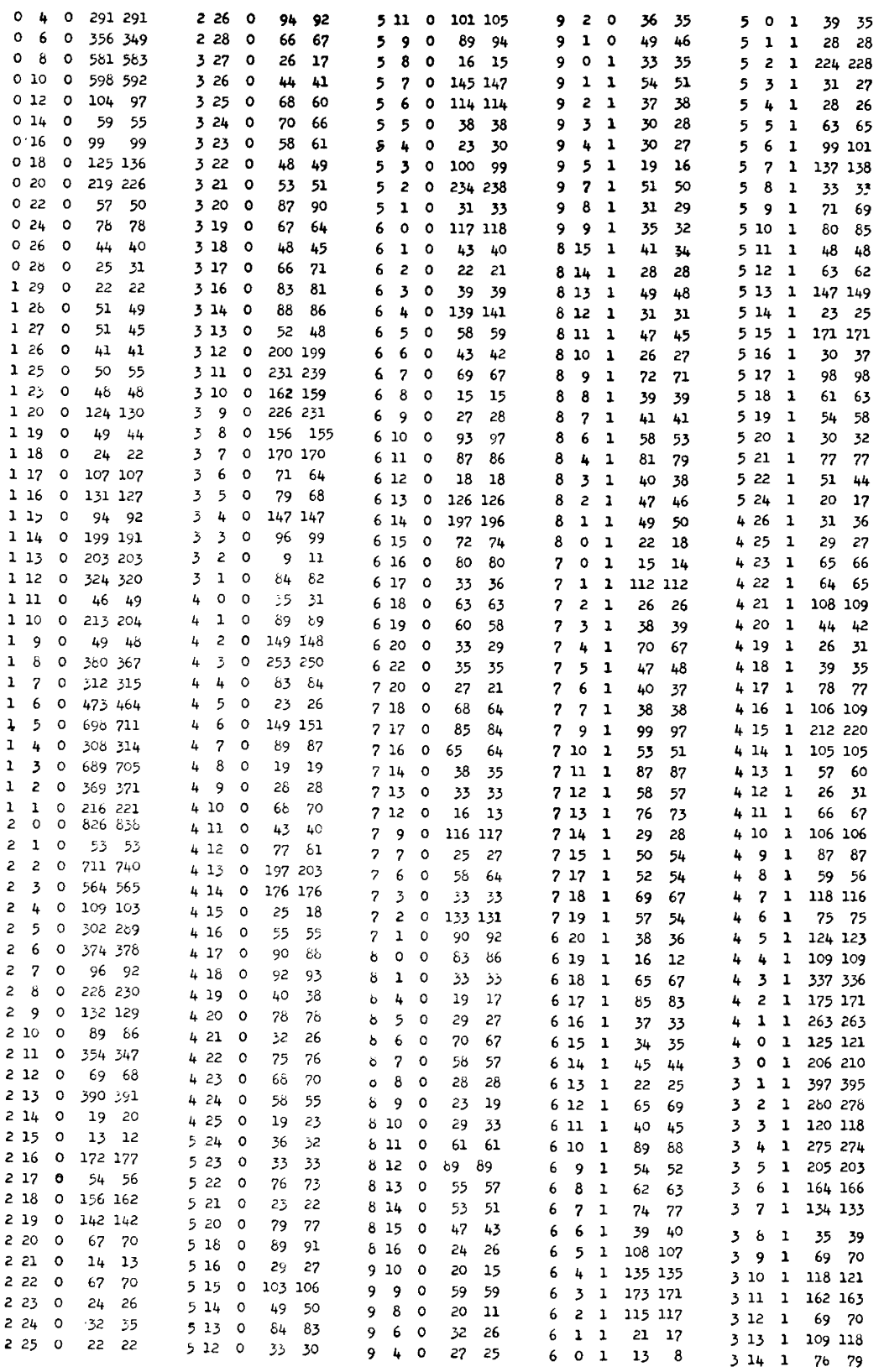


Table 1. Continued.

\begin{tabular}{|c|c|c|c|c|c|c|c|c|c|c|c|c|c|c|c|c|c|c|c|c|c|}
\hline 315 & 1 & 274 & 277 & & 24 & 1 & 25 & 26 & 1 & 3 & 2 & 314295 & 415 & 2 & 113 & 117 & 8 & & 2 & 69 & 71 \\
\hline 316 & 1 & 53 & 56 & & 23 & 1 & 60 & 54 & 2 & 2 & 2 & $493 \quad 468$ & 416 & 2 & 198 & 201 & 8 & 3 & 2 & 53 & 53 \\
\hline 317 & 1 & 51 & 56 & 0 & 22 & 1 & 57 & 56 & 1 & 1 & 2 & 293258 & 427 & 2 & 31 & 30 & 8 & 4 & 2 & 50 & 54 \\
\hline 318 & 2 & 63 & 63 & 0 & 21 & 1 & 61 & 65 & 1 & 0 & 2 & 366342 & 418 & 2 & 56 & 55 & 8 & 5 & 2 & 54 & 59 \\
\hline 319 & 1 & 38 & 39 & 0 & 20 & 1 & 48 & 48 & 2 & 0 & 2 & $57 \quad 63$ & 419 & 2 & 43 & 44 & 8 & 6 & 2 & 67 & 69 \\
\hline 320 & 1 & 75 & 76 & 0 & 19 & 1 & 105 & 113 & 2 & 1 & 2 & 220211 & 422 & 2 & 49 & 48 & 8 & 7 & 2 & 27 & 31 \\
\hline 322 & 1 & 40 & 39 & 0 & 18 & 1 & 76 & 81 & 2 & 2 & 2 & 124113 & 423 & 2 & 29 & 27 & 8 & 8 & 2 & 82 & 80 \\
\hline 323 & 1 & 64 & 62 & 0 & 17 & 1 & 109 & 108 & 2 & 3 & 2 & 453439 & 424 & 2 & 49 & 47 & 8 & 9 & 2 & 22 & 15 \\
\hline 324 & 1 & 57 & 55 & 0 & 16 & 1 & 19 & 26 & 2 & 4 & 2 & $213 \quad 205$ & 425 & 2 & 24 & 22 & 8 & 10 & 2 & 21 & 20 \\
\hline 325 & 2 & 35 & 30 & 0 & 15 & 1 & 54 & 61 & 2 & 5 & 2 & $232 \quad 223$ & 523 & 2 & 41 & 40 & 8 & 12 & 2 & 20 & 27 \\
\hline 326 & 1 & 29 & 29 & 0 & 14 & 1 & 131 & 132 & 2 & 6 & 2 & $168 \quad 162$ & 522 & 2 & 38 & 37 & 8 & 13 & 2 & 27 & 22 \\
\hline 327 & 1 & 36 & 35 & 0 & 13 & 1 & 21 & 22 & 2 & 7 & 2 & 154156 & 521 & 2 & 21 & 19 & 8 & 14 & 2 & 51 & 47 \\
\hline 228 & 1 & 57 & 52 & 0 & 11 & 1 & 228 & 223 & 2 & 8 & 2 & $42 \quad 41$ & 520 & 2 & 30 & 29 & 9 & 6 & 2 & 33 & 31 \\
\hline 227 & 2 & 24 & 23 & 0 & 10 & 1 & 230 & 222 & 2 & 9 & 2 & 74 & 519 & 2 & 73 & $7 ?$ & 9 & 4 & 2 & 21 & 22 \\
\hline 226 & 1 & 28 & 27 & 0 & 9 & 1 & 147 & 142 & 21 & 10 & 2 & 99101 & 518 & 2 & 25 & 22 & 9 & 3 & 2 & 65 & 64 \\
\hline 225 & 1 & 29 & 30 & 0 & 8 & 2 & 35 & 41 & 21 & 11 & 2 & $114 \quad 114$ & 517 & 2 & 40 & 39 & 9 & 2 & 2 & 30 & 28 \\
\hline 224 & 1 & 34 & 33 & 0 & 7 & 1 & 275 & 263 & 21 & 12 & 2 & $70 \quad 74$ & 516 & 2 & 20 & 23 & 9 & 1 & 2 & 27 & 29 \\
\hline 221 & 1 & 22 & 26 & 0 & 6 & 1 & 60 & 48 & 21 & 3 & 2 & 212218 & 515 & 2 & 1311 & 132 & 9 & 0 & 2 & 52 & 49 \\
\hline 220 & 1 & 57 & 52 & 0 & 5 & 1 & 230 & 220 & 21 & 4 & 2 & 144146 & 523 & 2 & 36 & 36 & 8 & 10 & 3 & 55 & 56 \\
\hline 219 & 1 & 61 & 65 & 0 & 3 & 1 & 255 & 252 & 21 & 15 & 2 & $148 \quad 155$ & 512 & 2 & 38 & 35 & 8 & 9 & 3 & 32 & 29 \\
\hline 218 & 1 & $1 \geqslant 8$ & 141 & 0 & 2 & 1 & 140 & 148 & 21 & 16 & 2 & $46 \quad 46$ & 511 & 2 & 1282 & 232 & 8 & 8 & 3 & 44 & 44 \\
\hline 217 & 1 & 146 & 149 & 0 & 2 & 1 & 339 & 294 & 21 & 7 & 2 & 173179 & 59 & 2 & 50 & 50 & 8 & $?$ & 3 & 67 & 66 \\
\hline 216 & 1 & 129 & 134 & 0 & 0 & 2 & 408 & 336 & 21 & 18 & 2 & 97101 & 5 & 2 & 31 & 30 & 8 & 6 & 3 & 59 & 60 \\
\hline 215 & 1 & 102 & 105 & 0 & 1 & 2 & 270 & 240 & 22 & 20 & 2 & 118119 & 5 & 2 & 74 & 76 & 8 & 5 & 3 & 48 & 46 \\
\hline 214 & 1 & 79 & 82 & 0 & 2 & 2 & 448 & 413 & 22 & 22 & 2 & $64 \quad 62$ & 5 & 2 & 2102 & 112 & 8 & 4 & 3 & 42 & 45 \\
\hline 213 & 1 & 111 & 112 & 0 & 3 & 2 & 50 & 35 & 22 & 3 & 2 & 20 & 5 & 2 & $188 \mathrm{I}$ & 186 & 8 & 2 & 3 & 20 & 24 \\
\hline 212 & 1 & 250 & 253 & 0 & 4 & 2 & 332 & 320 & 22 & & 2 & 36 & 5 & 2 & 219 & 226 & 8 & 1 & 3 & 55 & 54 \\
\hline 211 & 1 & 104 & 100 & 0 & 5 & 2 & 407 & 402 & 22 & 5 & 2 & 41 & 3 & 2 & 65 & 67 & 8 & 0 & 3 & 89 & 89 \\
\hline 210 & 1 & 275 & $26 ?$ & 0 & 6 & 2 & 323 & 305 & 22 & 6 & 2 & 44 & 2 & 2 & 69 & 67 & 7 & 0 & 3 & 100 & 101 \\
\hline 29 & 1 & 62 & 59 & 0 & 7 & 2 & 128 & 123 & 22 & $?$ & 2 & 70 & 1 & 2 & 1082 & 107 & 7 & 1 & 3 & 86 & 84 \\
\hline 8 & 1 & 121 & 121 & 0 & 8 & 2 & 78 & 84 & 32 & 6 & 2 & 48 & 0 & 2 & 19 & 16 & 7 & 2 & 3 & 130 & 130 \\
\hline 7 & 1 & 191 & 188 & 0 & 9 & 2 & 263 & 262 & 32 & 25 & 2 & 46 & 0 & 2 & 178 & 179 & 7 & 3 & 3 & 71 & 74 \\
\hline 6 & 1 & 236 & 225 & 0 & 10 & 2 & 55 & 58 & 32 & 24 & 2 & 23 & 2 & 2 & 62 & 61 & 7 & 4 & 3 & 97 & 99 \\
\hline 5 & 1 & 57 & 59 & 0 & 11 & 2 & 143 & 149 & 32 & 3 & 2 & 61 & 2 & 2 & 1261 & 130 & 7 & 5 & 3 & 26 & 28 \\
\hline 4 & 1 & 155 & 154 & 0 & 12 & 2 & 81 & 82 & 32 & 22 & 2 & 53 & 3 & 2 & 2082 & 213 & 7 & 6 & 3 & 75 & 74 \\
\hline 3 & 1 & 452 & 460 & 0 & 13 & 2 & 84 & 89 & 32 & & 2 & 39 & 4 & 2 & 67 & 67 & 7 & $?$ & 3 & 33 & 33 \\
\hline 2 & 1 & 311 & 309 & 0 & & 2 & 165 & 169 & 32 & & 2 & 47 & 5 & 2 & 63 & 65 & 7 & 8 & 3 & 50 & 52 \\
\hline 1 & 1 & 321 & 304 & 0 & 15 & 2 & 244 & 256 & 31 & & 2 & 74 & 6 & 2 & 75 & 78 & 7 & 9 & 3 & 50 & 49 \\
\hline 0 & 1 & 331 & 318 & 0 & 17 & 2 & 235 & 240 & 318 & 8 & 2 & 8689 & 7 & 2 & 34 & 39 & 7 & 10 & 3 & 22 & 18 \\
\hline 0 & 1 & 587 & 553 & 0 & 18 & 2 & 72 & 73 & 31 & & 2 & 135137 & 8 & 2 & 50 & 52 & 7 & 11 & 3 & 41 & 39 \\
\hline 1 & 1 & 694 & 671 & 0 & 21 & 2 & 52 & 53 & 316 & & 2 & 101105 & 69 & 2 & 56 & 59 & 7 & 12 & 3 & 85 & 83 \\
\hline 2 & 1 & 337 & 319 & 0 & 22 & 2 & 28 & 29 & 315 & & 2 & 135130 & 610 & 2 & 85 & 86 & 7 & 14 & 3 & 26 & 22 \\
\hline 3 & 1 & 56 & 51 & 0 & 23 & 2 & 74 & 74 & 314 & 4 & 2 & 123129 & 611 & 2 & 25 & 6 & 7 & 15 & 3 & 42 & 47 \\
\hline 4 & 2 & 402 & 391 & 0 & 24 & 2 & 54 & 53 & 31 & 3 & 2 & $110 \quad 212$ & 612 & 2 & 43 & 45 & $?$ & 16 & 3 & 21 & 20 \\
\hline 5 & 1 & 249 & 239 & 0 & 25 & 2 & 41 & 43 & 31 & & 2 & 118122 & 613 & 2 & 27 & 33 & 6 & 20 & 3 & 50 & 45 \\
\hline 6 & 1 & 123 & 117 & 0 & 26 & 2 & 36 & 32 & 310 & & 2 & 174178 & 614 & 2 & 32 & 34 & 6 & 19 & 3 & 31 & 36 \\
\hline 7 & 1 & 188 & 184 & 0 & 27 & 2 & 26 & 32 & 35 & 9 & 2 & 176176 & 615 & 2 & 44 & 41 & 6 & 18 & 3 & 78 & 75 \\
\hline 8 & 1 & 143 & 138 & 0 & 28 & 2 & 66 & 64 & 3 & 8 & 2 & $98 \quad 99$ & 626 & 2 & 66 & 64 & 6 & 17 & 3 & 21 & 17 \\
\hline 19 & 1 & 272 & 266 & 1 & 28 & 2 & 103 & 100 & 3 & 7 & 2 & 183160 & 617 & 2 & 54 & 54 & 6 & 16 & 3 & 30 & 30 \\
\hline 210 & 1 & 127 & 125 & 1 & 26 & 2 & 31 & 33 & 3 & 6 & 2 & 110102 & 618 & 2 & 47 & 41 & 6 & 15 & 3 & $4 ?$ & 44 \\
\hline 131 & 1 & 218 & 223 & 1 & 25 & 2 & 105 & 101 & 3 & 5 & 2 & 140141 & 619 & 2 & 34 & 33 & 6 & 14 & 3 & 26 & 25 \\
\hline 112 & 1 & 185 & 183 & 1 & 24 & 2 & 36 & 34 & 3 & 4 & 2 & 211206 & 620 & 2 & 42 & 37 & 6 & 12 & 3 & 61 & 66 \\
\hline 113 & 1 & 174 & 177 & 1 & 23 & 2 & 54 & 52 & 3 & 3 & 2 & $228 \quad 222$ & 621 & 2 & 59 & 5? & 6 & 11 & 3 & 31 & 33 \\
\hline 114 & 1 & 179 & 181 & 1 & 22 & 2 & 44 & 42 & 3 & 2 & 2 & 292286 & 718 & 2 & 29 & 29 & 6 & 10 & 3 & 80 & 80 \\
\hline 215 & 1 & 80 & 83 & 1 & 20 & 2 & 21 & 25 & 3 & 1 & 2 & 145139 & 727 & 2 & $4 ?$ & 44 & 6 & 9 & 3 & 64 & 63 \\
\hline 116 & 1 & 55 & 59 & 1 & 19 & 2 & 24 & 28 & 3 & 0 & 2 & 229224 & 716 & 2 & 24 & 20 & 6 & 8 & 3 & 15 & 24 \\
\hline 117 & 1 & 140 & 241 & 1 & 18 & 2 & 213 & 219 & 4 & 0 & 2 & $36 \quad 39$ & 715 & 2 & 21 & 18 & 6 & 7 & 3 & 29 & 30 \\
\hline 118 & 1 & 53 & $5 ?$ & 1 & 17 & 2 & 78 & 82 & 4 & 1 & 2 & 210213 & 714 & 2 & 30 & 29 & 6 & 6 & 3 & 94 & 93 \\
\hline 119 & 1 & 104 & 107 & 1 & 16 & 2 & 131 & 136 & 4 & 2 & 2 & 144146 & 713 & 2 & 22 & 25 & 6 & 5 & 3 & 15 & 15 \\
\hline 120 & 1 & 52 & 57 & 1 & 15 & 2 & 180 & 187 & 4 & 3 & 2 & $89 \quad 89$ & 712 & 2 & 59 & 59 & 6 & 4 & 3 & 64 & 62 \\
\hline 121 & 1 & 79 & 83 & 1 & 14 & 2 & 143 & 146 & 4 & 4 & 2 & $\begin{array}{ll}72 \quad 72\end{array}$ & 711 & 2 & 68 & 66 & 6 & 3 & 3 & 21 & 24 \\
\hline 222 & 1 & 15 & 21 & 1 & 13 & 2 & 98 & 103 & 4 & 5 & 2 & 173170 & 710 & 2 & 32 & 35 & 6 & 2 & 3 & 89 & 88 \\
\hline 1.24 & 1 & 36 & 40 & 1 & 12 & 2 & 77 & 78 & 4 & 6 & 2 & 142144 & 79 & 2 & 30 & 29 & 6 & 1 & 3 & 67 & 69 \\
\hline 125 & 1 & 40 & 38 & 1 & 12 & 2 & 27 & 14 & 4 & 7 & 2 & 6366 & 78 & 2 & 77 & 80 & 6 & 0 & 3 & 151 & 152 \\
\hline 126 & 1 & 72 & $6 ?$ & 1 & 10 & 2 & 66 & 64 & 4 & 8 & 2 & 156158 & 7 & 2 & 51 & 54 & 5 & 0 & 3 & 117 & 121 \\
\hline 128 & 1 & 59 & 51 & 1 & 9 & 2 & 110 & 105 & 4 & 9 & 2 & $120 \quad 118$ & 4 & 2 & 79 & 78 & 5 & 1 & 3 & 95 & 95 \\
\hline 029 & $i$ & 20 & 25 & 1 & 8 & 2 & 302 & 303 & 420 & & 2 & 208113 & 3 & 2 & 36 & 39 & 3 & 2 & 3 & 244 & 244 \\
\hline 028 & 1 & 69 & 62 & 1 & 7 & 2 & 154 & 253 & 42 & & 2 & $\begin{array}{ll}69 & 74\end{array}$ & 1 & 2 & 57 & 58 & 5 & 3 & 3 & 94 & 94 \\
\hline 027 & 1 & 33 & 31 & 1 & 62 & 2 & 299 & 298 & 412 & & 2 & $28 \quad 29$ & 0 & 2 & 150 & 154 & 5 & 4 & 3 & 81 & 86 \\
\hline 026 & 1 & 51 & 49 & 1 & 5 & 2 & 386 & 372 & 42 & & 2 & $136 \quad 139$ & 0 & 2 & 84 & 81 & 5 & 5 & 3 & 40 & 40 \\
\hline 025 & 1 & 43 & 42 & 1 & 4 & 2 & 169 & 154 & 414 & & 2 & 156162 & 81 & 2 & 53 & 53 & 5 & 6 & 3 & 98 & 99 \\
\hline
\end{tabular}


Table 1. Continued.

\begin{tabular}{|c|c|c|c|c|}
\hline 5 & 7 & 3 & 77 & 77 \\
\hline 5 & 8 & 3 & 36 & 35 \\
\hline 5 & 9 & 3 & 19 & $a$ \\
\hline 510 & & 3 & 88 & 9 \\
\hline 51 & & 3 & 97 & 98 \\
\hline 51 & & 3 & 40 & 41 \\
\hline & & $\begin{array}{l}3 \\
3\end{array}$ & $\begin{array}{l}78 \\
22\end{array}$ & $\begin{array}{l}78 \\
24\end{array}$ \\
\hline 51 & & 3 & 51 & 50 \\
\hline 51 & & 3 & 94 & 90 \\
\hline 52 & & 3 & 30 & 34 \\
\hline 42 & & 3 & 46 & 45 \\
\hline 422 & & 3 & 50 & 47 \\
\hline 421 & & 3 & 46 & $4 ?$ \\
\hline 420 & & 3 & 87 & 88 \\
\hline 419 & & 3 & 29 & 26 \\
\hline 418 & & 3 & 84 & 84 \\
\hline 417 & & 3 & 50 & 53 \\
\hline 416 & & 3 & 16 & 14 \\
\hline 415 & & 3 & 87 & 89 \\
\hline 414 & & 3 & 36 & 32 \\
\hline 413 & & 3 & 83 & 86 \\
\hline 412 & & $\begin{array}{ll}37 \\
\end{array}$ & 70 & 75 \\
\hline 410 & & 312 & 231 & 129 \\
\hline 49 & 3 & 38 & 81 & 88 \\
\hline 48 & 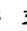 & 33 & 36 & 34 \\
\hline 47 & 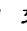 & 320 & $? 2$ & 210 \\
\hline 46 & & 3 & 15 & 75 \\
\hline 4 & & 3112 & 41 & 113 \\
\hline $4 \quad 4$ & . & 310 & 7 & 10 \\
\hline 43 & . & 3150 & & 151 \\
\hline 42 & 3 & 380 & 0 & 80 \\
\hline 41 & 3 & 393 & 3 & 99 \\
\hline 40 & 3 & 3190 & & 195 \\
\hline 30 & 3 & 362 & & 68 \\
\hline 32 & 3 & 3241 & & 34 \\
\hline 32 & 3 & 349 & +95 & 57 \\
\hline 33 & 3 & $3 \quad 137$ & 37 & 30 \\
\hline 34 & 3 & 58 & 88 & 57 \\
\hline 35 & 3 & 68 & 86 & 68 \\
\hline 36 & 3 & 159 & 916 & 60 \\
\hline 37 & 3 & 162 & 216 & 65 \\
\hline 38 & 3 & 89 & 98 & 82 \\
\hline 39 & 3 & 117 & 712 & 120 \\
\hline 310 & 3 & & 28 & 83 \\
\hline 311 & 3 & & 18 & 84 \\
\hline 312 & 3 & 132 & & 33 \\
\hline 313 & 3 & 120 & & 23 \\
\hline 314 & 3 & 105 & & 07 \\
\hline 315 & 3 & 111 & & 14 \\
\hline 316 & 3 & 117 & 12 & 22 \\
\hline 327 & 3 & 67 & & 70 \\
\hline 318 & 3 & 98 & 810 & 01 \\
\hline 319 & 3 & 77 & & 75 \\
\hline 320 & 3 & 36 & & $3 ?$ \\
\hline 321 & 3 & 96 & & 95 \\
\hline 322 & 3 & 36 & & 35 \\
\hline 323 & 3 & 52 & & 49 \\
\hline 324 & 3 & 57 & & 57 \\
\hline 325 & 3 & 20 & & 9 \\
\hline 226 & 3 & 61 & & 57 \\
\hline 224 & 3 & 24 & & 26 \\
\hline 223 & 3 & 46 & & 46 \\
\hline 222 & 3 & 61 & & 63 \\
\hline 221 & 3 & 104 & 102 & 22 \\
\hline 220 & 3 & 30 & 29 & 29 \\
\hline 219 & 3 & 134 & +137 & 57 \\
\hline 218 & 3 & 115 & 5118 & 18 \\
\hline 217 & 3 & 28 & & 28 \\
\hline 216 & 3 & 127 & 132 & \\
\hline 215 & 3 & 54 & 55 & \\
\hline
\end{tabular}

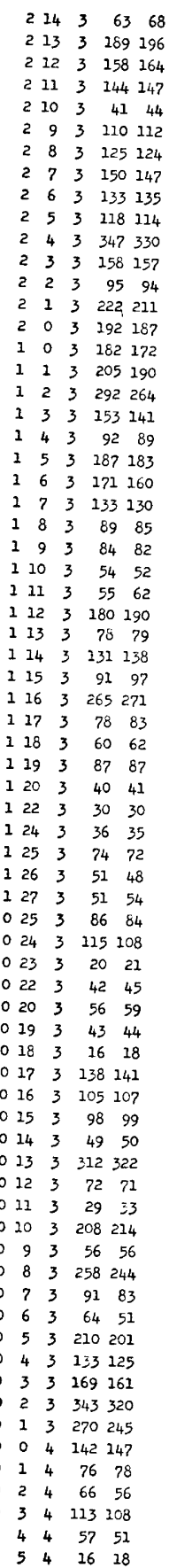

$\begin{array}{lllll}0 & 6 & 4 & 170 & 175\end{array}$ $\begin{array}{lllll}0 & 8 & 4 & 113 & 118\end{array}$ $\begin{array}{lllll}0 & 10 & 4 & 17 & 11\end{array}$ $\begin{array}{lllll}0 & 11 & 4 & 25 & 28\end{array}$ $\begin{array}{lllll}0 & 12 & 4 & 58 & 64\end{array}$ $\begin{array}{llllll}0 & 13 & 4 & 168 & 176\end{array}$ $\begin{array}{llll}0 & 14 & 4 & 50 \quad 46\end{array}$ $\begin{array}{lllll}0 & 15 & 4 & 43 & 43\end{array}$ $\begin{array}{lllll}0 & 16 & 4 & 34 & 33\end{array}$ $\begin{array}{lllll}0 & 17 & 4 & 43 & 24\end{array}$ $\begin{array}{lllll}0 & 18 & 4 & 25 & 26\end{array}$ $\begin{array}{llll}0 & 19 \quad 4 \quad 25 & 27\end{array}$ $\begin{array}{llll}0 & 20 & 4 & 47\end{array} 46$ $\begin{array}{lllll}0 & 21 & 4 & 101 & 96\end{array}$ $\begin{array}{lllll}0 & 23 \quad 4 \quad 46 & 16\end{array}$ $\begin{array}{lllll}0 & 24 & 4 & 65 & 66\end{array}$ $\begin{array}{lllll}1 & 25 & 4 & 34 & 36\end{array}$ $\begin{array}{lllll}1 & 24 & 4 & 39 & 36\end{array}$ $\begin{array}{lllll}1 & 23 & 4 & 50 & 49\end{array}$ $\begin{array}{lllll}1 & 22 & 4 & 112 & 111\end{array}$ $\begin{array}{lllll}1 & 21 & 4 & 37 & 35\end{array}$ $\begin{array}{lllll}1 & 20 & 4 & 31 & 34\end{array}$ $\begin{array}{lllll}1 & 19 & 4 & 20 & 15\end{array}$ $\begin{array}{lllll}1 & 18 & 4 & 39 & 44\end{array}$ $\begin{array}{lllll}1 & 17 & 4 & 49 & 50\end{array}$ $\begin{array}{lllll}1 & 16 & 4 & 81 & 83\end{array}$ $\begin{array}{lllll}1 & 15 & 4 & 67 & 92\end{array}$ $\begin{array}{lllll}2 & 14 & 4 & 27 & 30\end{array}$

$\begin{array}{lllll}1 & 13 & 4 & 112 & 119\end{array}$

$\begin{array}{lllll}1 & 12 & 4 & 361 & 165\end{array}$

$\begin{array}{lllll}1 & 11 & 4 & 78 & 84\end{array}$

$120 \quad 4 \quad 1410$

$\begin{array}{lllll}1 & 9 & 4 & 137 & 137\end{array}$

$\begin{array}{lllll}1 & 8 & 4 & 120 & 133\end{array}$ $\begin{array}{lllll}1 & 7 & 4 & 23 & 23\end{array}$ $\begin{array}{lllll}1 & 6 & 4 & 72 & 60\end{array}$ $\begin{array}{lllll}1 & 5 & 4 & 71 & 72\end{array}$ $144 \quad 112114$ 1334180179 $\begin{array}{lllll}1 & 2 & 4 & 114 & 108\end{array}$ $11145243 \quad 230$ $\begin{array}{lllll}1 & 0 & 4 & 178 & 172\end{array}$ $\begin{array}{lllll}2 & 0 & 4 & 113 & 109\end{array}$ $\begin{array}{lllll}2 & 1 & 4 & 81 & 86\end{array}$ $\begin{array}{lllll}2 & 2 & 4 & 89 & 94\end{array}$ $\begin{array}{lllll}2 & 3 & 4 & 199 & 195\end{array}$ $244 \quad 135135$ 2 $54 \quad 120121$ $\begin{array}{lllll}2 & 6 & 4 & 46 & 47\end{array}$ $\begin{array}{llllll}2 & 7 & 4 & 27 & 30\end{array}$ $\begin{array}{lllll}2 & 8 & 4 & 100 & 95\end{array}$ 2945150 $210 \quad 4 \quad 102110$ $\begin{array}{lllll}211 & 4 & 122 & 130\end{array}$ $\begin{array}{lllll}2 & 12 & 4 & 89 & 92\end{array}$ $\begin{array}{lllll}2 & 13 & 4 & 80 & 83\end{array}$ $\begin{array}{lllll}2 & 14 & 4 & 19 & 20\end{array}$

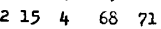
$\begin{array}{lllll}2 & 16 & 4 & 56 & 59\end{array}$ $\begin{array}{lllll}2 & 17 & 4 & 99 & 99\end{array}$ $\begin{array}{lllll}2 & 18 & 4 & 47 & 48\end{array}$ $\begin{array}{lllll}2 & 19 & 4 & 60 & 65\end{array}$ $\begin{array}{lllll}2 & 20 & 4 & 44 & 46\end{array}$ $\begin{array}{llll}21 & 4 & 69 & 68\end{array}$ $\begin{array}{lllll}22 & 4 & 28 & 22\end{array}$ $\begin{array}{lllll}2 & 24 & 4 & 20 & 21\end{array}$ $\begin{array}{lllll}3 & 23 & 4 & 47 & 48\end{array}$ $\begin{array}{lllll}3 & 21 & 4 & 66 & 63\end{array}$ $\begin{array}{lllll}320 & 4 & 35 & 28\end{array}$ $\begin{array}{lllll}3 & 19 & 4 & 63 & 61\end{array}$ $318 \quad 4 \quad 110112$ $\begin{array}{lllll}3 & 17 & 4 & 82 & 83\end{array}$ $\begin{array}{lllll}3 & 16 & 4 & 89 & 91\end{array}$ $\begin{array}{lllll}3 & 15 & 4 & 45 & 46\end{array}$ $\begin{array}{llllll}3 & 14 & 4 & 135 & 138\end{array}$ $\begin{array}{lllll}3 & 13 & 4 & 139 & 146\end{array}$ $\begin{array}{lllll}3 & 12 & 4 & 151 & 156\end{array}$ $\begin{array}{lllll}3 & 11 & 4 & 97 & 101\end{array}$

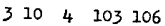
$\begin{array}{lllll}3 & 9 & 4 & 126 & 128\end{array}$ $\begin{array}{lllll}3 & 8 & 4 & 36 & 37\end{array}$ $\begin{array}{lllll}3 & 7 & 4 & 71 & 67\end{array}$ $\begin{array}{lllll}3 & 6 & 4 & 122 & 127\end{array}$ $\begin{array}{lllll}3 & 5 & 4 & 31 & 30\end{array}$ $\begin{array}{lllll}3 & 4 & 4 & 79 & 85\end{array}$ $\begin{array}{llllll}3 & 3 & 4 & 106 & 107\end{array}$ $\begin{array}{lllll}3 & 2 & 4 & 257 & 255\end{array}$ $\begin{array}{lllll}3 & 1 & 4 & 134 & 132\end{array}$ $\begin{array}{llllll}3 & 0 & 4 & 140 & 136\end{array}$ $\begin{array}{lllll}4 & 0 & 4 & 99 & 101\end{array}$ $\begin{array}{lllll}4 & 1 & 4 & 175 & 178\end{array}$ $\begin{array}{lllll}4 & 2 & 4 & 128 & 127\end{array}$ $\begin{array}{lllll}4 & 3 & 4 & 27 & 34\end{array}$ $\begin{array}{lllll}4 & 4 & 4 & 25 & 29\end{array}$ $\begin{array}{lllll}4 & 5 & 4 & 82 & 89\end{array}$ $\begin{array}{lllll}4 & 6 & 4 & 51 & 57\end{array}$ $\begin{array}{lllll}4 & 7 & 4 & 98 & 100\end{array}$ $\begin{array}{lllll}4 & 8 & 4 & 65 & 66\end{array}$ $\begin{array}{lllll}4 & 9 & 4 & 77 & 80\end{array}$ $\begin{array}{lllll}410 & 4 & 129 & 135\end{array}$

$\begin{array}{llll}411 & 4 & 70 & 67\end{array}$

$\begin{array}{lllll}4 & 12 & 4 & 33 & 34\end{array}$

$\begin{array}{lllll}4 & 13 & 4 & 76 & 78\end{array}$

$\begin{array}{lllll}4 & 14 & 4 & 30 & 30\end{array}$

$\begin{array}{lllll}4 & 15 & 4 & 29 & 27\end{array}$

$\begin{array}{lllll}426 & 4 & 42 & 43\end{array}$

$\begin{array}{lllll}4 & 19 & 4 & 59 & 61\end{array}$

$\begin{array}{lllll}420 & 4 & 45 & 43\end{array}$

$\begin{array}{lllll}4 & 21 & 4 & 26 & 19\end{array}$

$\begin{array}{lllll}4222 & 4 & 40 & 40\end{array}$

$\begin{array}{lllll}520 & 4 & 85 & 85\end{array}$

$\begin{array}{lllll}5 & 19 & 4 & 107 & 107\end{array}$

$\begin{array}{lllll}5 & 17 & 4 & 21 & 25\end{array}$ $\begin{array}{lllll}5 & 16 & 4 & 39 & 39\end{array}$ $\begin{array}{lllll}5 & 15 & 4 & 36 & 35\end{array}$ $\begin{array}{lllll}5 & 14 & 4 & 21 & 17\end{array}$ $\begin{array}{lllll}513 & 4 & 62 & 66\end{array}$ $\begin{array}{lllll}5 & 12 & 4 & 39 & 40\end{array}$ $\begin{array}{lllll}5 & 11 & 4 & 135 & 138\end{array}$ $\begin{array}{lllll}5 & 10 & 4 & 43 & 48\end{array}$ $\begin{array}{lllll}5 & 9 & 4 & 66 & 68\end{array}$ $\begin{array}{lllll}5 & 8 & 4 & 89 & 91\end{array}$ $\begin{array}{lllll}5 & 7 & 4 & 19 & 20\end{array}$ $\begin{array}{lllll}5 & 6 & 4 & 51 & 51\end{array}$ $\begin{array}{lllll}5 & 5 & 4 & 77 & 82\end{array}$ $\begin{array}{lllll}5 & 4 & 4 & 23 & 27\end{array}$ $\begin{array}{lllll}5 & 3 & 4 & 52 & 55\end{array}$ $\begin{array}{lllll}5 & 2 & 4 & 65 & 64\end{array}$ $\begin{array}{lllll}5 & 1 & 4 & 142 & 145\end{array}$ $\begin{array}{lllll}5 & 0 & 4 & 204 & 205\end{array}$ $\begin{array}{lllll}6 & 0 & 4 & 65 & 69\end{array}$ $\begin{array}{lllll}6 & 1 & 4 & 94 & 99\end{array}$ $\begin{array}{lllll}6 & 2 & 4 & 47 & 49\end{array}$ $\begin{array}{lllll}6 & 3 & 4 & 23 & 27\end{array}$ $\begin{array}{lllll}6 & 4 & 4 & 29 & 31\end{array}$ $\begin{array}{lllll}6 & 5 & 4 & 29 & 28\end{array}$ $\begin{array}{lllll}6 & 6 & 4 & 57 & 57\end{array}$ $\begin{array}{lllll}6 & 7 & 4 & 28 & 25\end{array}$ $\begin{array}{lllll}6 & 8 & 4 & 55 & 55\end{array}$ $\begin{array}{lllll}6 & 9 & 4 & 41 & 45\end{array}$ $\begin{array}{lllll}6 & 10 & 4 & 24 & 23\end{array}$ $\begin{array}{lllll}6 & 11 & 4 & 33 & 31\end{array}$ $\begin{array}{lllll}6 & 12 & 4 & 29 & 31\end{array}$

$\begin{array}{lllll}6 & 13 & 4 & 48 & 51\end{array}$

$\begin{array}{lllll}6 & 15 & 4 & 67 & 64\end{array}$

$\begin{array}{lllll}6 & 16 & 4 & 36 & 36\end{array}$

$\begin{array}{lllll}6 & 17 & 4 & 32 & 30\end{array}$

$\begin{array}{lllll}7 & 12 & 4 & 25 & 28\end{array}$

$\begin{array}{lllll}7 & 11 & 4 & 28 & 29\end{array}$

$\begin{array}{lllll}7 & 10 & 4 & 21 & 18\end{array}$

$\begin{array}{llllll}7 & 9 & 4 & 7 ? & 74\end{array}$

$\begin{array}{lllll}7 & 8 & 4 & 56 & 56\end{array}$

$\begin{array}{lllll}7 & 7 & 4 & 44 & 42\end{array}$

$\begin{array}{lllll}7 & 6 & 4 & 31 & 31\end{array}$

$\begin{array}{lllll}7 & 5 & 4 & 70 & 67\end{array}$

$\begin{array}{lllll}7 & 3 & 4 & 39 & 41\end{array}$

$\begin{array}{lllll}7 & 2 & 4 & 48 & 48\end{array}$

$\begin{array}{lllll}7 & 1 & 4 & 52 & 52\end{array}$

$\begin{array}{lllll}8 & 0 & 4 & 22 & 20\end{array}$

$\begin{array}{lllll}8 & 1 & 4 & 49 & 46\end{array}$

$\begin{array}{lllll}8 & 3 & 4 & 45 & 39\end{array}$

$\begin{array}{lllll}8 & 4 & 4 & 32 & 35\end{array}$ $\begin{array}{lllll}8 & 5 & 4 & 37 & 38\end{array}$ $\begin{array}{lllll}8 & 6 & 4 & 18 & 11\end{array}$ $\begin{array}{lllll}8 & 7 & 4 & 22 & 17\end{array}$ $\begin{array}{lllll}7 & 0 & 5 & 65 & 65\end{array}$ $\begin{array}{lllll}7 & 1 & 5 & 55 & 56\end{array}$ $\begin{array}{lllll}7 & 2 & 5 & 31 & 27\end{array}$ $\begin{array}{lllll}7 & 3 & 5 & 58 & 57\end{array}$ $\begin{array}{lllll}7 & 4 & 5 & 31 & 31\end{array}$ $75542 \quad 43$ $\begin{array}{lllll}7 & 6 & 5 & 31 & 32\end{array}$ $\begin{array}{lllll}7 & 8 & 5 & 31 & 32\end{array}$ $\begin{array}{lllll}6 & 13 & 5 & 69 & 66\end{array}$ $\begin{array}{lllll}6 & 12 & 5 & 20 & 26\end{array}$

$\begin{array}{llll}611 & 5 & 70 & 75\end{array}$ $\begin{array}{lllll}6 & 10 & 5 & 52 & 5\end{array}$ $\begin{array}{lllll}6 & 9 & 5 & 56 & 55\end{array}$ $\begin{array}{lllll}6 & 8 & 5 & 35 & 38\end{array}$ $\begin{array}{lllll}6 & 6 & 5 & 62 & 60\end{array}$ $\begin{array}{lllll}6 & 5 & 5 & 81 & 60\end{array}$ $\begin{array}{lllll}6 & 4 & 5 & 88 & 86\end{array}$

$\begin{array}{lllll}6 & 3 & 5 & 26 & 19\end{array}$

$\begin{array}{lllll}6 & 2 & 5 & 56 & 57\end{array}$

$\begin{array}{lllll}6 & 1 & 5 & 27 & 26\end{array}$

$\begin{array}{lllll}6 & 0 & 5 & 36 & 36\end{array}$

$\begin{array}{lllll}5 & 0 & 5 & 117 & 120\end{array}$

$\begin{array}{lllll}5 & 1 & 5 & 60 & 58\end{array}$

$\begin{array}{lllll}5 & 2 & 5 & 42 & 38\end{array}$

$\begin{array}{lllll}5 & 3 & 5 & 41 & 42\end{array}$

$\begin{array}{lllll}5 & 4 & 5 & 25 & 26\end{array}$

$\begin{array}{lllll}5 & 5 & 5 & 26 & 22\end{array}$

$\begin{array}{lllll}5 & 6 & 5 & 54 & 53\end{array}$

$\begin{array}{lllll}5 & 7 & 5 & 62 & 62\end{array}$

$\begin{array}{lllll}5 & 8 & 5 & 65 & 64\end{array}$ $\begin{array}{lllll}5 & 9 & 5 & 86 & 86\end{array}$ $\begin{array}{lllll}5 & 10 & 5 & 24 & 29\end{array}$ $\begin{array}{lllll}5 & 11 & 5 & 35 & 37\end{array}$ $\begin{array}{lllll}5 & 12 & 5 & 67 & 70\end{array}$ $\begin{array}{lllll}5 & 13 & 5 & 57 & 55\end{array}$ $\begin{array}{lllll}5 & 14 & 5 & 51 & 48\end{array}$ $\begin{array}{lllll}515 & 5 & 45 & 45\end{array}$ $\begin{array}{lllll}5 & 16 & 5 & 37 & 33\end{array}$ $\begin{array}{lllll}4 & 19 & 5 & 25 & 24\end{array}$ $\begin{array}{lllll}4 & 18 & 5 & 80 & 79\end{array}$ $\begin{array}{lllll}417 & 5 & 32 & 35\end{array}$ $\begin{array}{lllll}4 & 16 & 5 & 88 & 80\end{array}$

$\begin{array}{lllll}4 & 15 & 4 & 75 & 75\end{array}$

$\begin{array}{lllll}4 & 14 & 5 & 31 & 37\end{array}$

$\begin{array}{lllll}4 & 13 & 5 & 52 & 58\end{array}$

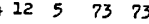

$\begin{array}{rrrr}411 & 5 & 98 & 101 \\ 410 & 5 & 81 & 82\end{array}$ 
Table 1. Continued.

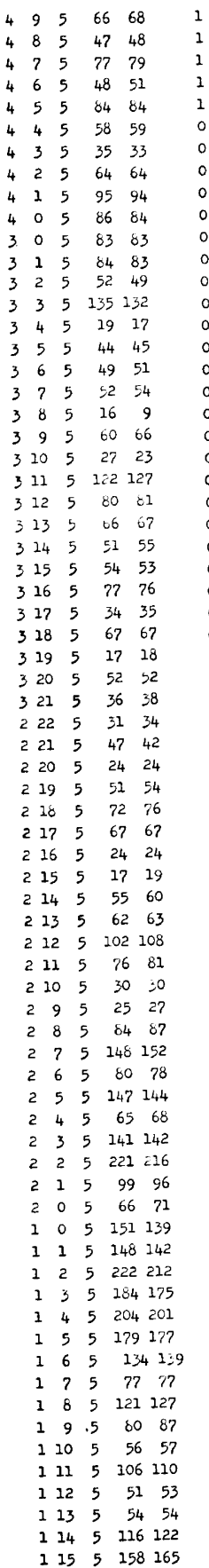

\begin{tabular}{|c|c|c|c|}
\hline 116 & 5 & 22 & 24 \\
\hline 118 & 5 & 70 & 73 \\
\hline 119 & 5 & 44 & 46 \\
\hline 121 & 5 & 45 & 44 \\
\hline 122 & 5 & 49 & 47 \\
\hline 023 & 5 & 31 & 34 \\
\hline 022 & 5 & 23 & 20 \\
\hline 21 & 5 & 90 & 90 \\
\hline 20 & 5 & 47 & 46 \\
\hline 19 & 5 & 61. & 61 \\
\hline 18 & 5 & 94 & 94 \\
\hline I? & 5 & 32 & 31 \\
\hline 16 & 5 & 45 & 51 \\
\hline 15 & 5 & 30 & 31 \\
\hline 013 & 5 & 100 & 107 \\
\hline 011 & 5 & 88 & 87 \\
\hline 010 & 5 & 68 & 66 \\
\hline 9 & 5 & 211 & 226 \\
\hline 8 & 5 & 162 & 2164 \\
\hline 7 & 5 & 69 & $\begin{array}{l}9 \\
9\end{array}$ \\
\hline 6 & 5 & 146 & 6147 \\
\hline 5 & 5 & $4 ?$ & $7 \quad 46$ \\
\hline 4 & 5 & 129 & 9126 \\
\hline 3 & 5 & 40 & $0 \quad 40$ \\
\hline 2 & 5 & 213 & 3207 \\
\hline 1 & 5 & 277 & 7268 \\
\hline 00 & 6 & 42 & 246 \\
\hline 02 & 6 & 66 & 60 \\
\hline 3 & 6 & 101 & 1 96 \\
\hline 4 & 6 & 163 & 160 \\
\hline 5 & 6 & 20 & $10 \quad 29$ \\
\hline 6 & 6 & 7 & 175 \\
\hline$?$ & 6 & 9 & 194 \\
\hline 8 & 6 & & 4651 \\
\hline 09 & 6 & & 16 \\
\hline 010 & 6 & & 20 \\
\hline 011 & 6 & & $23 \quad 20$ \\
\hline 012 & 6 & & 33 \\
\hline 013 & 6 & & $16 \quad 11$ \\
\hline 014 & 6 & & $\begin{array}{ll}72 & 74\end{array}$ \\
\hline 015 & 6 & 13 & 33142 \\
\hline 016 & 6 & & 3643 \\
\hline 0219 & 6 & & $21 \quad 14$ \\
\hline 119 & 6 & & $47 \quad 49$ \\
\hline 118 & 36 & & $37 \quad 32$ \\
\hline 117 & 76 & & $57 \quad 55$ \\
\hline 116 & 66 & & $45 \quad 43$ \\
\hline 115 & 56 & & $52 \quad 50$ \\
\hline 114 & 46 & & $30 \quad 38$ \\
\hline 112 & 26 & 6 & $42 \quad 43$ \\
\hline 111 & 16 & 6 & $30 \quad 28$ \\
\hline 110 & 06 & 6 & $43 \quad 44$ \\
\hline 29 & 96 & & 5760 \\
\hline 18 & 86 & & 98101 \\
\hline 1 & 76 & 6 & $40 \quad 39$ \\
\hline 16 & 66 & 6 & $30 \quad 30$ \\
\hline 1 & 56 & 6 & $65 \quad 63$ \\
\hline 14 & 4 & 6 & 8382 \\
\hline 1 & 3 & 6 & 155152 \\
\hline 1 & 26 & 6 & $41 \quad 34$ \\
\hline 1 & 1 & 6 & $50 \quad 47$ \\
\hline 2 & 0 & 6 & $20 \quad 23$ \\
\hline 2 & 1 & 6 & $54 \quad 53$ \\
\hline 2 & 2 & 6 & $26 \quad 29$ \\
\hline 2 & 3 & 6 & 6162 \\
\hline 2 & 4 & 6 & 120120 \\
\hline 2 & 5 & 6 & 6363 \\
\hline 2 & 6 & 6 & $73 \quad 74$ \\
\hline 2 & $?$ & 6 & $18 \quad 19$ \\
\hline 2 & 8 & 6 & $74 \quad 80$ \\
\hline 2 & 9 & 6 & $61 \quad 62$ \\
\hline
\end{tabular}

\begin{tabular}{|c|c|c|c|}
\hline 210 & 6 & $27 \quad 29$ & 29 \\
\hline 211 & 6 & 242 & 25 \\
\hline 212 & 6 & 403 & 39 \\
\hline 213 & 6 & 505 & 52 \\
\hline 2. 14 & 6 & 424 & 47 \\
\hline 215 & 6 & 323 & 30 \\
\hline 216 & 6 & 555 & 57 \\
\hline 217 & 6 & 53 & 55 \\
\hline 218 & 6 & 424 & 40 \\
\hline 317 & 6 & 465 & 51 \\
\hline 316 & 6 & 25 & 25 \\
\hline 315 & 6 & 76 & 76 \\
\hline 314 & 6 & 41 & 34 \\
\hline 312 & 6 & 41 & 42 \\
\hline 311 & 6 & 64 & 63 \\
\hline 310 & 6 & 26 & 25 \\
\hline 39 & 6 & 50 & 49 \\
\hline 38 & 6 & 72 & 73 \\
\hline 37 & 6 & 40 & 40 \\
\hline 36 & 6 & 1231 & 123 \\
\hline 5 & 6 & 66 & 67 \\
\hline 34 & 6 & 68 & 62 \\
\hline 33 & 6 & 1031 & 106 \\
\hline 32 & 6 & 25 & 71 \\
\hline 31 & 6 & 24 & 24 \\
\hline 40 & 6 & 57 & 56 \\
\hline 41 & 6 & 22 & 19 \\
\hline 43 & 6 & 53 & 55 \\
\hline 44 & 6 & 36 & 31 \\
\hline 45 & 6 & 68 & 72 \\
\hline 46 & 6 & 43 & 45 \\
\hline 47 & 6 & 71 & 66 \\
\hline 49 & 6 & 25 & 30 \\
\hline 410 & 6 & 46 & 46 \\
\hline 431 & 6 & 31 & 29 \\
\hline 422 & 6 & 36 & 35 \\
\hline 413 & 6 & 33 & 33 \\
\hline 414 & 6 & 76 & 79 \\
\hline 415 & 6 & 42 & 40 \\
\hline 512 & 6 & 42 & 45 \\
\hline 510 & 6 & 19 & 23 \\
\hline 59 & 6 & 61 & 60 \\
\hline 58 & 6 & 46 & 42 \\
\hline 56 & 6 & 123 & 122 \\
\hline 55 & 6 & 31 & 23 \\
\hline 54 & 6 & 92 & 91 \\
\hline 53 & 6 & 48 & 43 \\
\hline 50 & 6 & 98 & 97 \\
\hline 61 & 6 & 79 & 78 \\
\hline 62 & 6 & 43 & 48 \\
\hline 63 & 6 & 40 & 38 \\
\hline 64 & 46 & 56 & 53 \\
\hline 65 & 56 & 44 & 42 \\
\hline $\begin{array}{ll}6 & 6 \\
6 & 7\end{array}$ & $\begin{array}{ll}6 & 6 \\
7 & 6\end{array}$ & $\begin{array}{l}27 \\
55\end{array}$ & $\begin{array}{l}28 \\
53\end{array}$ \\
\hline 48 & 87 & 30 & 28 \\
\hline 47 & 77 & 65 & 68 \\
\hline 46 & 67 & 74 & 73 \\
\hline 45 & 57 & $7 ?$ & 77 \\
\hline 44 & 47 & 58 & $5 ?$ \\
\hline 43 & 37 & 61 & 61 \\
\hline 4 & 27 & 20 & 15 \\
\hline 4 & 07 & 122 & 121 \\
\hline 3 & 07 & 74 & 73 \\
\hline 3 & 17 & 45 & 42 \\
\hline 3 & 27 & 47 & 44 \\
\hline 3 & $3 ?$ & 45 & 45 \\
\hline 3 & 47 & 38 & 36 \\
\hline 3 & 57 & 21 & 72 \\
\hline 3 & 67 & 76 & 76 \\
\hline 3 & 87 & 18 & 18 \\
\hline
\end{tabular}

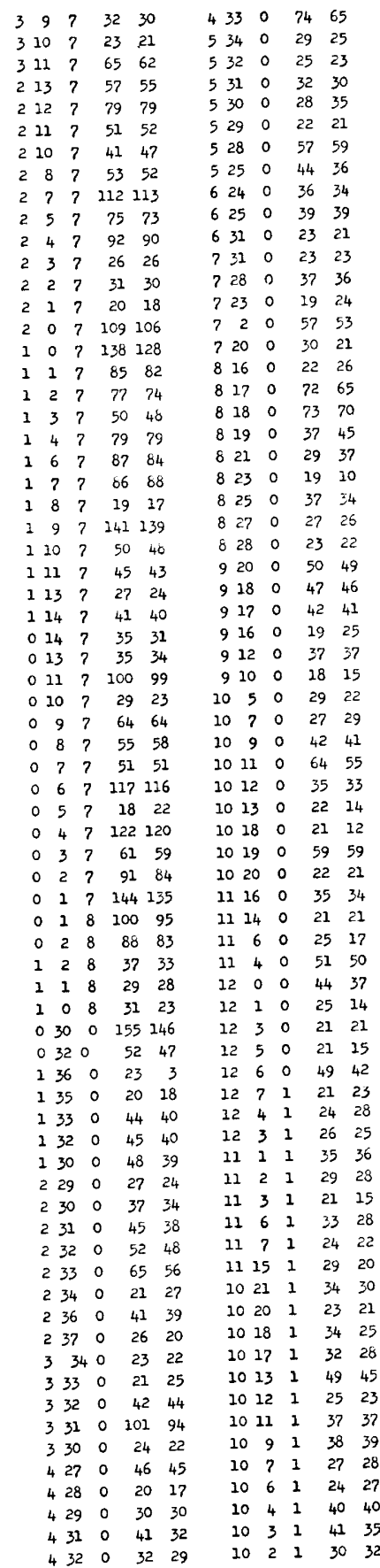


Table 1. Continued.

\begin{tabular}{|c|c|c|c|c|c|c|c|c|c|c|c|c|c|c|c|c|c|c|c|c|c|c|c|}
\hline 10 & 0 & 1 & 37 & 32 & & 32 & 2 & 20 & 24 & 10 & & 2 & 29 & 23 & 529 & 3 & 36 & 32 & & 26 & 4 & 38 & 31 \\
\hline 9 & 9 & 1 & 32 & 32 & 0 & & 2 & 34 & 40 & 10 & 12 & 2 & 20 & 11 & 530 & 3 & 55 & 50 & & 25 & 4 & 21 & \\
\hline & 10 & 2 & 23 & 21 & 0 & & 2 & 44 & 39 & 10 & 13 & 2 & 36 & 35 & 532 & 3 & 32 & 23 & & 23 & 4 & 28 & \\
\hline 9 & 11 & 1 & 33 & 35 & 0 & 36 & 2 & 29 & 26 & 10 & 14 & 2 & 23 & 21 & 433 & 3 & 35 & 29 & 5 & 22 & 4 & 35 & \\
\hline 9 & & 1 & 73 & 67 & 0 & 37 & 2 & 32 & 30 & 11 & 14 & 2 & 21 & 12 & 432 & 3 & 36 & 28 & & 21 & 4 & 56 & \\
\hline 9 & 18 & 1 & 45 & 45 & 1 & 36 & 2 & 44 & 42 & 11 & 13 & 2 & 22 & 21 & 431 & 3 & 31 & 27 & & 18 & 4 & 25 & \\
\hline 9 & 19 & 1 & 24 & 19 & & 34 & 2 & 42 & 38 & 11 & 9 & 2 & 30 & 34 & 429 & 3 & 33 & 31 & 6 & 19 & 4 & 38 & \\
\hline 9 & 20 & 2 & 23 & 27 & 1 & 30 & 2 & 38 & 36 & 11 & 8 & 2 & 31 & 26 & 428 & 3 & 36 & 37 & 6 & 20 & 4 & 29 & \\
\hline 9 & 21 & 1 & 34 & 33 & & 28 & 2 & 37 & 32 & 11 & 6 & 2 & 23 & 18 & 427 & 3 & 46 & 45 & 6 & 21 & 4 & 51 & \\
\hline 8 & 28 & 1 & 23 & 24 & 2 & 30 & 2 & 86 & 82 & 11 & 3 & 2 & 31 & 28 & 426 & 3 & 19 & 14 & 6 & 23 & 4 & 38 & \\
\hline 8 & 26 & 1 & 20 & 26 & 2 & 31 & 2 & 52 & 51 & 11 & 2 & 2 & 28 & 24 & 425 & 3 & 23 & 27 & 6 & 24 & 4 & 29 & \\
\hline 8 & 21 & 1 & 24 & 21 & 2 & 33 & 2 & 27 & 25 & 11 & 1 & 2 & 20 & 18 & 326 & 3 & 25 & 31 & 6 & 25 & 4 & 22 & \\
\hline 8 & 19 & 1 & 31 & 32 & 2 & 36 & 2 & 23 & 14 & 11 & 0 & 2 & 32 & 27 & 328 & 3 & 18 & 12 & 6 & 26 & 4 & 20 & \\
\hline 8 & 17 & 1 & 36 & 35 & 3 & 35 & 2 & 22 & 13 & 12 & 2 & 2 & 26 & 23 & 330 & 3 & 43 & 41 & 6 & 27 & 4 & 22 & \\
\hline 8 & 16 & 1 & 73 & 71 & 3 & 34 & 2 & 29 & 21 & 11 & 5 & 3 & 24 & 24 & 331 & 3 & 31 & 28 & 6 & 28 & 4 & 25 & \\
\hline 7 & 21 & 1 & 30 & 23 & 3 & 33 & 2 & 27 & 30 & 11 & 11 & 3 & 23 & 16 & 332 & 3 & 26 & 26 & 6 & 29 & 4 & 45 & \\
\hline 7 & 23 & 1 & 19 & 17 & 3 & 31 & 2 & 37 & 32 & 10 & 19 & 3 & 23 & 21 & 333 & 3 & 22 & 22 & 7 & 26 & 4 & 45 & \\
\hline$?$ & 24 & 1 & 44 & 42 & 3 & 30 & 2 & 29 & 29 & 10 & 18 & 3 & 22 & 20 & 235 & 3 & 23 & 15 & $?$ & 25 & 4 & 36 & \\
\hline 7 & 26 & 1 & 20 & 13 & 3 & 29 & 2 & 21 & 17 & 10 & 15 & 3 & 20 & 14 & 234 & 3 & 24 & 23 & 7 & 22 & 4 & 28 & \\
\hline 7 & 27 & 1 & 20 & 16 & 3 & 28 & 2 & 65 & 55 & 10 & 14 & 3 & 24 & 16 & 233 & 3 & 42 & 40 & 7 & 21 & 4 & 36 & \\
\hline 7 & 28 & 1 & 39 & 36 & & 27 & 2 & 48 & 50 & 10 & 13 & 3 & 51 & 48 & 231 & 3 & 36 & 32 & 7 & 19 & 4 & 53 & \\
\hline 7 & 29 & 1 & 31 & 23 & 4 & 26 & 2 & 19 & 21 & 10 & 9 & 3 & 22 & 5 & 229 & 3 & 71 & 67 & 7 & 18 & 4 & 41 & \\
\hline 6 & 31 & 1 & 22 & 20 & 4 & 27 & 2 & 54 & 48 & 10 & 7 & 3 & 21 & 28 & 228 & 3 & 29 & 30 & 7 & 17 & 4 & 42 & \\
\hline 6 & 30 & 2 & 20 & 22 & 4 & 28 & 2 & 50 & $4 ?$ & 10 & 6 & 3 & 51 & 49 & 127 & 3 & 51 & 54 & 7 & 26 & 4 & 46 & \\
\hline 6 & 28 & 1 & 38 & 35 & 4 & 30 & 2 & 39 & 32 & 10 & 4 & 3 & 49 & 48 & 128 & 3 & 31 & 32 & 7 & 15 & 4 & 38 & \\
\hline 6 & 27 & 1 & 45 & 44 & 4 & 31 & 2 & 49 & 43 & 10 & 3 & 3 & 23 & 21 & 129 & 3 & 51 & 47 & $?$ & 14 & 4 & 30 & \\
\hline 6 & 26 & 1 & 39 & 41 & 5 & 29 & 2 & 22 & 18 & 10 & 2 & 3 & 21 & 21 & 130 & 3 & 27 & 28 & 8 & 7 & 4 & 19 & \\
\hline 6 & 24 & 1 & 73 & 74 & 5 & 28 & 2 & 35 & 32 & 10 & 1 & 3 & 23 & 14 & 131 & 3 & 19 & 3 & 8 & 8 & 4 & 20 & \\
\hline 5 & 25 & 1 & 50 & 46 & 5 & 26 & 2 & 45 & 45 & 9 & 0 & 3 & 46 & 48 & 135 & 3 & 34 & 35 & 8 & 10 & 4 & 24 & 3 \\
\hline 5 & 26 & 1 & 25 & 26 & 5 & 24 & 2 & 29 & 27 & 9 & 1 & 3 & 43 & 42 & 136 & 3. & 26 & 26 & 8 & 12 & 4 & 35 & \\
\hline 5 & 27 & 1 & 30 & 34 & 6 & 24 & 2 & 28 & 29 & 9 & 2 & 3 & 25 & 34 & 035 & 3 & 22 & 22 & 8 & 13 & 4 & 42 & 37 \\
\hline 5 & 28 & 1 & $4 ?$ & 46 & 6 & 26 & 2 & 46 & 6 & 9 & 3 & 3 & 27 & 23 & 033 & 3 & 35 & 28 & 8 & 14 & 4 & 50 & \\
\hline 5 & 29 & 1 & 35 & 32 & 6 & 27 & 2 & 67 & 62 & 9 & 4 & 3 & 44 & 51 & 032 & 3 & 80 & 76 & 8 & 15 & 4 & 30 & \\
\hline 5 & 30 & 1 & 27 & 25 & 6 & 28 & 2 & 40 & 35 & 9 & 5 & 3 & 52 & 53 & 031 & 3 & 46 & 49 & 8 & 17 & 4 & 37 & \\
\hline 5 & 32 & 1 & 38 & 36 & 6 & 29 & 2 & 22 & 14 & 9 & 6 & 3 & 48 & 52 & 030 & 3 & 53 & 50 & 8 & 18 & 4 & 24 & \\
\hline 3 & 33 & 1 & 30 & 26 & 6 & 31 & 2 & 25 & 23 & 9 & 8 & 3 & 26 & 29 & 029 & 3 & 34 & 35 & 8 & 20 & 4 & 24 & \\
\hline 4 & 35 & 1 & 24 & 23 & $?$ & 28 & 2 & 25 & 20 & 9 & 10 & 3 & 27 & 27 & 028 & 3 & 29 & 24 & 8 & 24 & 4 & 24 & \\
\hline 4 & 33 & 1 & 24 & 21 & 7 & 26 & 2 & 34 & 32 & 9 & 11 & 3 & 20 & 20 & 026 & 4 & 24 & 18 & 9 & 20 & 4 & 33 & \\
\hline 4 & 32 & 1 & 29 & 30 & $?$ & 23 & 2 & 27 & 30 & 9 & 14 & 3 & 40 & 35 & 028 & 4 & 21 & 29 & 9 & 18 & 4 & 34 & 34 \\
\hline 4 & 31 & 1 & 42 & 39 & 7 & 22 & 2 & 42 & 37 & 9 & 15 & 3 & 26 & 33 & 030 & 4 & 52 & 49 & 9 & 17 & 4 & 38 & \\
\hline 4 & 30 & 1 & 44 & 38 & 7 & 21 & 2 & 18 & 16 & 9 & 16 & 3 & 28 & 26 & 034 & 4 & 29 & 29 & 9 & 14 & 4 & 22 & \\
\hline 4 & 29 & 1 & 24 & 25 & 7 & 20 & 2 & 37 & 38 & 9 & 19 & 3 & 21 & 18 & 134 & 4 & 25 & 19 & 9 & 13 & 4 & 45 & 37 \\
\hline 4 & 27 & 1 & 40 & 38 & 7 & 19 & 2 & 45 & 42 & 9 & 20 & 3 & 29 & 27 & 133 & 4 & 67 & 60 & 9 & 12 & 4 & 54 & \\
\hline 3 & 28 & 1 & 37 & 35 & 8 & 15 & 2 & 22 & 26 & 9 & 21 & 3 & 25 & 19 & 131 & 4 & 53 & 47 & 9 & 10 & 4 & 22 & \\
\hline 3 & 29 & 2 & 49 & 49 & 8 & 16 & 2 & 64 & 62 & 9 & 22 & 3 & 34 & 24 & 130 & 4 & 33 & 33 & 9 & 8 & 4 & 19 & 26 \\
\hline 3 & 30 & 1 & 27 & 31 & 8 & 17 & 2 & 24 & 20 & 8 & 26 & 3 & 39 & 33 & 128 & 4 & 21 & 26 & 9 & $?$ & 4 & 41 & \\
\hline 3 & 31 & 1 & 33 & 30 & 8 & 18 & 2 & 50 & 49 & 8 & 22 & 3 & 21 & 10 & 127 & 4 & 38 & 36 & 9 & 6 & 4 & 31 & \\
\hline 3 & 32 & 1 & 37 & 34 & 8 & 19 & 2 & 20 & 28 & 8 & 20 & 3 & 28 & 28 & 126 & 4 & 39 & 42 & 9 & 5 & 4 & 52 & \\
\hline 3 & 33 & 1 & 42 & 41 & 8 & 22 & 2 & 38 & 36 & 8 & 17 & 3 & 21 & 19 & 225 & 4 & 32 & 35 & 9 & 4 & 4 & 38 & 88 \\
\hline 3 & 34 & 1 & 33 & 24 & 8 & 24 & 2 & 20 & 14 & 8 & 15 & 3 & 64 & 63 & 227 & 4 & 30 & 33 & 9 & 3 & 4 & 48 & 49 \\
\hline 2 & 34 & 1 & 24 & 18 & 8 & 26 & 2 & 22 & 17 & 8 & 13 & 3 & 41 & 41 & 228 & 4 & 23 & 26 & 9 & 2 & 4 & 22 & 18 \\
\hline 2 & 33 & 1 & 23 & 27 & 8 & 27 & 2 & 27 & 23 & 8 & 12 & 3 & 29 & 24 & 234 & 4 & 25 & 30 & 9 & 0 & 4 & 21 & 26 \\
\hline 2 & 32 & 1 & 42 & 33 & 9 & 23 & 2 & 21 & 16 & 7 & 18 & 3 & 40 & 38 & 333 & 4 & 37 & 27 & 10 & 2 & 4 & 23 & \\
\hline 2 & 31 & 1 & 22 & 28 & 9 & 21 & 2 & 28 & 25 & 7 & 19 & 3 & 19 & 26 & 332 & 4 & 25 & 27 & 10 & 4 & 4 & 28 & \\
\hline 2 & 30 & 1 & 52 & 44 & 9 & 20 & 2 & 26 & 27 & 7 & 20 & 3 & 29 & 17 & 331 & 4 & 34 & 33 & 10 & 6 & 4 & 19 & 16 \\
\hline 2 & 29 & 1 & 42 & 38 & 9 & 19 & 2 & 24 & 19 & 7 & 23 & 3 & 21 & 5 & 330 & 4 & 28 & 28 & 10 & 7 & 4 & 25 & \\
\hline 1 & 29 & 1 & 84 & 74 & 9 & 17 & 2 & 33 & 35 & 7 & 24 & 3 & 37 & 33 & 328 & 4 & 31 & 27 & 10 & 12 & 4 & 30 & \\
\hline 1 & 30 & 1 & 28 & 30 & 9 & 16 & 2 & 22 & 21 & 7 & 26 & 3 & 23 & 22 & 327 & 4 & 27 & 27 & 10 & 14 & 4 & 23 & 18 \\
\hline 1 & 31 & 1 & 73 & 69 & 9 & 14 & 2 & 42 & 41 & 7 & 28 & 3 & 31 & 26 & 325 & 4 & 22 & 26 & 10 & 15 & 4 & 47 & 48 \\
\hline 1 & 33 & 1 & 33 & 27 & 9 & 13 & 2 & 65 & 64 & 7 & 29 & 3 & 30 & 25 & 324 & 4 & 59 & 58 & 10 & 26 & 4 & 23 & 20 \\
\hline 1 & 34 & 1 & 23 & 21 & 9 & 12 & 2 & 38 & 32 & 6 & 26 & 3 & 51 & 47 & 423 & 4 & 40 & 44 & 11 & 9 & 4 & 30 & 23 \\
\hline 1 & 35 & 1 & 26 & 18 & 9 & 11 & 2 & 34 & 35 & 6 & 25 & 3 & 29 & 35 & 424 & 4 & 25 & 22 & 11 & 7 & 4 & 28 & \\
\hline 1 & 37 & 1 & 22 & 24 & 9 & 10 & 2 & 46 & 49 & 6 & 23 & 3 & 21 & 20 & 425 & 4 & 39 & 37 & 11 & 6 & 4 & 40 & \\
\hline 0 & 34 & 1 & 43 & 34 & 9 & 9 & 2 & 54 & 53 & 6 & 22 & 3 & 29 & 26 & 426 & 4 & 21 & 16 & 11 & 5 & 4 & 33 & \\
\hline 0 & 32 & 1 & 39 & 32 & 9 & 8 & 2 & 25 & 23 & 6 & 21 & 3 & 17 & 10 & 428 & 4 & 31 & 23 & 11 & 4 & 4 & 21 & \\
\hline 0 & 31 & 1 & 24 & 19 & 10 & 0 & 2 & 58 & 62 & 6 & 20 & 3 & 48 & 45 & 429 & 4 & 31 & 27 & 11 & 3 & 4 & 30 & \\
\hline 0 & 30 & 1 & 57 & 52 & 10 & 2 & 2 & 29 & 31 & 5 & 23 & 3 & 28 & 25 & 430 & 4 & 42 & 41 & 11 & 1 & 4 & 22 & \\
\hline 0 & 29 & 1 & $21^{\circ}$ & 25 & 10 & 3 & 2 & 25 & 23 & 5 & 25 & 3 & 22 & 24 & 432 & 4 & $4 ?$ & 44 & 20 & 10 & 5 & 26 & \\
\hline 0 & 29 & 2 & 40 & 42 & 10 & 4 & 2 & 41 & 43 & 5 & 26 & 3 & 43 & 40 & 531 & 4 & 26 & 26 & 10 & $?$ & 5 & 26 & \\
\hline 0 & 30 & 2 & 50 & 44 & 10 & 5 & 2 & 60 & 58 & 5 & 27 & 3 & 32 & 33 & 530 & 4 & 42 & 45 & 10 & 6 & 5 & 27 & \\
\hline
\end{tabular}


Table 1. Continued.

\begin{tabular}{|c|c|c|c|c|c|c|c|c|c|c|c|c|c|c|c|c|c|c|c|c|c|}
\hline 10 & 2 & 5 & 33 & 34 & 224 & 5 & 30 & 33 & 61 & & 6 & 36 & 35 & 8 & 7 & 55 & 55 & 214 & 7 & 97 & 98 \\
\hline 9 & 3 & 5 & 29 & 26 & 223 & 5 & 63 & 60 & 61 & 6 & 6 & 50 & 48 & 79 & 7 & 25 & 21 & 213 & 7 & 59 & 55 \\
\hline 9 & 4 & 5 & 36 & 33 & 222 & 5 & 33 & 34 & 61 & 17 & 6 & 21 & 24 & 711 & $?$ & 24 & 16 & 114 & 7 & 36 & 40 \\
\hline 9 & 7 & 5 & 24 & 19 & 123 & 5 & 22 & 20 & 61 & 28 & 6 & 49 & 50 & 712 & 7 & 30 & 29 & 115 & 7 & 34 & 35 \\
\hline 9 & 8 & 5 & 19 & 8 & 124 & 5 & 54 & 57 & 61 & 19 & 6 & 26 & 18 & 714 & 7 & 27 & 13 & 117 & 7 & 57 & 62 \\
\hline 9 & 9 & 5 & 33 & 31 & 125 & 5 & 19 & 25 & 62 & 22 & 6 & 22 & 19 & 215 & 7 & 21 & 18 & 118 & $?$ & 53 & 52 \\
\hline 91 & 11 & 5 & 37 & 38 & 126 & 5 & 69 & 66 & 62 & 23 & 6 & 37 & 34 & 716 & 7 & 34 & 34 & 119 & $?$ & 58 & 60 \\
\hline 9 & 14 & 5 & 41 & 43 & 127 & 5 & 32 & 28 & 72 & 22 & 6 & 24 & 18 & ? 18 & 7 & 33 & 35 & 120 & 7 & 60 & 64 \\
\hline 9 & 15 & 5 & 47 & 46 & 128 & 5 & 25 & 18 & 72 & 20 & 6 & 33 & 37 & 619 & 7 & 28 & 30 & 121 & 7 & 32 & 35 \\
\hline 9 & 16 & 5 & 34 & 34 & 129 & 5 & 31 & 26 & 71 & & 6 & 37 & 37 & 618 & 7 & 27 & 25 & 122 & 7 & 31 & 32 \\
\hline 9 & 18 & 5 & 24 & 15 & 130 & 5 & 28 & 32 & 71 & & 6 & 36 & 33 & 614 & 7 & 26 & 28 & 123 & 7 & 25 & 16 \\
\hline 8 & 21 & 5 & 26 & 27 & 132 & 5 & 30 & 26 & 71 & & 6 & 29 & 32 & 613 & $?$ & 50 & 46 & 124 & 7 & 23 & 24 \\
\hline 8 & 20 & 5 & 29 & 21 & 029 & 5 & 47 & 47 & 71 & & 6 & 33 & 24 & 611 & $?$ & 51 & 49 & 125 & 7 & 38 & 34 \\
\hline 8 & 29 & 5 & 22 & 17 & 028 & 5 & 48 & 46 & 71 & 14 & 6 & 26 & 28 & 610 & 7 & 33 & 27 & 126 & 7 & 27 & 13 \\
\hline 8 & 17 & 5 & 58 & 57 & 024 & 5 & 36 & 37 & 71 & & 6 & 42 & 37 & 69 & 7 & 51 & 47 & 12 ? & 7 & 47 & 46 \\
\hline 8 & 16 & 5 & 53 & 49 & 023 & 5 & 36 & 34 & 71 & 12 & 6 & 24 & 16 & 6 & $?$ & 22 & 24 & 026 & 7 & 52 & 50 \\
\hline 8 & 14 & 5 & 24 & 19 & 020 & 6 & 32 & 33 & 71 & 11 & 6 & 30 & 27 & 7 & $?$ & 47 & 47 & 025 & 7 & 23 & 25 \\
\hline 8 & 13 & 5 & 35 & 36 & 023 & 6 & 39 & 38 & 71 & & 6 & 70 & 74 & 6 & 7 & 29 & 27 & 023 & 7 & 43 & 43 \\
\hline$\delta$ & 9 & 5 & 59 & 59 & 026 & 6 & 65 & 59 & $?$ & 9 & 6 & 22 & 27 & 4 & $?$ & 23 & 21 & 022 & 7 & 32 & 28 \\
\hline 8 & 7 & 5 & 26 & 28 & 028 & 6 & 53 & 50 & 7 & 8 & 6 & 26 & 28 & 3 & 7 & 64 & 62 & 021 & 7 & 27 & 18 \\
\hline 8 & 5 & 5 & 30 & 31 & 129 & 6 & 41 & 36 & 7 & 7 & 6 & 40 & 40 & 6 & 7 & 24 & 24 & 020 & $?$ & 29 & 32 \\
\hline 8 & 4 & 5 & 28 & 30 & 128 & 6 & 20 & 11 & 7 & 6 & 6 & 45 & 45 & 5 & 7 & 25 & 23 & 019 & 7 & 87 & 87 \\
\hline 8 & 3 & 5 & 24 & 26 & 127 & 6 & 53 & 50 & $?$ & 5 & 6 & 28 & 23 & 5 & 7 & 54 & 26 & 017 & 7 & 67 & 61 \\
\hline o & 2 & 5 & 36 & 40 & 126 & 6 & 33 & 32 & $?$ & 4 & 6 & 23 & 22 & 5 & $?$ & 35 & 33 & 03 & 8 & 19 & 15 \\
\hline$?$ & 8 & 5 & 33 & 32 & 120 & 6 & 42 & 42 & 7 & 2 & 6 & 69 & 70 & 5 & 7 & 96 & 97 & 6 & 8 & 28 & 24 \\
\hline 7 & 9 & 5 & 70 & 68 & 219 & 6 & 29 & 32 & 7 & 1 & 6 & 42 & 41 & 5 & 7 & 63 & 67 & 7 & 8 & 28 & 30 \\
\hline 7 & 10 & 5 & 32 & 32 & 220 & 6 & 45 & 43 & 7 & 0 & 6 & 68 & 63 & 58 & 7 & 35 & 33 & 8 & 8 & 57 & 54 \\
\hline 73 & 11 & 5 & 50 & 52 & 221 & 6 & 28 & 29 & 8 & 0 & 6 & 19 & 11 & 510 & 7 & 28 & 27 & 09 & 8 & 73 & 71 \\
\hline 7 & 12 & 5 & 40 & 40 & 222 & 6 & 41 & 39 & 8 & 2 & 6 & 28 & 21 & 511 & 7 & 35 & 32 & 010 & 8 & 1031 & 101 \\
\hline 7 & 13 & 5 & 46 & 48 & 224 & 6 & 21 & 29 & 8 & 3 & 6 & 60 & 61 & 512 & 7 & 45 & 42 & 011 & 8 & 2191 & 122 \\
\hline$?$ & 15 & 5 & 49 & 47 & 226 & 6 & 53 & 48 & 8 & 4 & 6 & 35 & 36 & 513 & 7 & 69 & 69 & O 14 & 8 & 24 & 23 \\
\hline 7 & 16 & 5 & 46 & 46 & 228 & 6 & 50 & 48 & 8 & 5 & 6 & 48 & 49 & 514 & 7 & 51 & 54 & 025 & 8 & 37 & 37 \\
\hline 7 & 17 & 5 & 34 & 39 & 229 & 6 & 23 & 21 & 8 & 6 & 6 & 23 & 16 & 517 & 7 & 42 & 46 & 016 & 8 & 43 & 44 \\
\hline 7 & 19 & 5 & 44 & 46 & 329 & 6 & 26 & 27 & 8 & 7 & 6 & 30 & 20 & 520 & $?$ & 22 & 16 & 019 & 8 & 32 & 36 \\
\hline 7 & 22 & 5 & 21 & 22 & 325 & 6 & 24 & 26 & 8 & 9 & 6 & 35 & 28 & 425 & $?$ & 49 & 44 & 021 & 8 & 81 & 82 \\
\hline 7 & 23 & 5 & 25 & 22 & 324 & 6 & 49 & 47 & 81 & 10 & 6 & 52 & 54 & 424 & 7 & 28 & 26 & 024 & 8 & 32 & 27 \\
\hline 7 & 25 & 5 & 25 & 28 & 323 & 6 & 45 & 44 & 81 & 11 & 6 & 20 & 19 & 422 & 7 & 30 & 28 & 122 & 8 & 31 & 26 \\
\hline 6 & 25 & 5 & 24 & 10 & 321 & 6 & 25 & 25 & 81 & 12 & 6 & 20 & 22 & 421 & 7 & 25 & 26 & 121 & 8 & 43 & 43 \\
\hline 6 & 23 & 5 & 20 & 16 & 320 & 6 & 39 & 36 & 83 & 14 & 6 & 41 & 38 & 419 & 7 & 25 & 21 & 120 & 8 & 48 & 50 \\
\hline 6 & 21 & 5 & 29 & 31 & 319 & 6 & 83 & 84 & 82 & 15 & 6 & 29 & 29 & 418 & 7 & 54 & 53 & 119 & 8 & 38 & 39 \\
\hline 6 & 20 & 5 & 63 & 67 & 318 & 6 & 58 & 59 & 83 & 16 & 6 & 37 & 38 & 416 & 7 & 37 & 40 & 118 & 8 & 68 & 68 \\
\hline 6 & 19 & 5 & 20 & 27 & 416 & 6 & 41 & 41 & 83 & 17 & 6 & 22 & 17 & 415 & 7 & 32 & 32 & 116 & 8 & 46 & 49 \\
\hline 6 & 17 & 5 & 49 & 48 & 417 & 6 & 75 & 74 & 83 & 18 & 6 & 24 & 22 & 424 & 7 & 79 & 81 & 115 & 8 & 28 & 26 \\
\hline 6 & 16 & 5 & 42 & 46 & 418 & 6 & 32 & 31 & 93 & 12 & 6 & 30 & 25 & 412 & $?$ & 27 & 24 & 113 & 8 & 27 & 28 \\
\hline 6 & 15 & 5 & 43 & 37 & 420 & 6 & 39 & 39 & 9 & 11 & 6 & 27 & 26 & 411 & 7 & 62 & 65 & 112 & 8 & 58 & 58 \\
\hline 5 & 18 & 5 & 38 & 32 & 421 & 6 & 37 & 37 & 9 & 8 & 6 & 26 & 27 & 49 & 7 & 32 & 34 & 110 & 8 & 83 & 81 \\
\hline 5 & 19 & 5 & 53 & 57 & 422 & 6 & 24 & 29 & 9 & 6 & 6 & 27 & 21 & 48 & 7 & 31 & 28 & 19 & 8 & 20 & 21 \\
\hline 5 & 21 & 5 & 22 & 20 & 424 & 6 & 44 & 42 & 9 & 5 & 6 & 26 & 21 & 311 & 7 & 63 & 62 & 1 & 8 & 69 & 71 \\
\hline 5 & 22 & 5 & 37 & 36 & 425 & 6 & 25 & 21 & 9 & 3 & 6 & 28 & 24 & 312 & $?$ & 84 & 85 & 1 & 8 & 35 & 36 \\
\hline 5 & 28 & 5 & 42 & 34 & 426 & 6 & 24 & 21 & 9 & 1 & 6 & 20 & 15 & 313 & 7 & 54 & $5 ?$ & 1 & 8 & 21 & 23 \\
\hline 5 & 29 & 5 & 28 & 23 & 427 & 6 & 23 & 24 & 10 & 0 & 6 & 27 & 28 & 314 & 7 & 40 & 38 & 1 & 8 & 43 & 43 \\
\hline 4 & 30 & 5 & 21 & 20 & 428 & 6 & 22 & 19 & 10 & 2 & 6 & 28 & 24 & 316 & 7 & 30 & 31 & 2 & 8 & 45 & 40 \\
\hline 4 & 26 & 5 & 44 & 40 & 526 & 6 & 42 & 39 & 9 & 0 & 7 & 37 & 32 & 317 & 7 & 24 & 27 & 1 & 8 & 27 & 33 \\
\hline 4 & 24 & 5 & 37 & 37 & 524 & 6 & 22 & 13 & 9 & 1 & 7 & 35 & 35 & 318 & 7 & 62 & 61 & 1 & 8 & 30 & 28 \\
\hline 4 & 23 & 5 & 40 & 37 & 523 & 6 & 30 & 29 & 9 & 2 & 7 & 30 & 20 & 319 & 7 & 43 & 43 & 2 & 8 & 26 & 23 \\
\hline 4 & 22 & 5 & 42 & 40 & 521 & 6 & 34 & $3 ?$ & 9 & 3 & 7 & 29 & 30 & 320 & 7 & 52 & 54 & 2 & 8 & 21 & 23 \\
\hline 4 & 21 & 5 & 31 & 31 & 520 & 6 & 24 & 25 & 9 & 4 & 7 & 23 & 16 & 322 & 7 & 45 & 43 & 2 & 8 & 33 & 74 \\
\hline 4 & 20 & 5 & 65 & 66 & 518 & 6 & 25 & 22 & 8 & 21 & 7 & 23 & 22 & 325 & $?$ & 25 & 24 & 2 & 8 & 56 & 55 \\
\hline 3 & 21 & 5 & 38 & 38 & 517 & 6 & 73 & 70 & 8 & 7 & $?$ & 31 & 30 & 326 & 7 & 38 & 36 & 2 & 8 & 54 & 57 \\
\hline 3 & 22 & 5 & $6 ?$ & 66 & 516 & 6 & 75 & 74 & 8 & 6 & 7 & 24 & 17 & 227 & 7 & 33 & 27 & 2 & 8 & 27 & 23 \\
\hline 3 & 23 & 5 & 35 & 38 & 515 & 6 & 57 & 57 & 8 & 5 & 7 & 28 & 23 & 225 & 7 & 32 & $2 ?$ & 25 & 8 & 57 & 54 \\
\hline 3 & 24 & 5 & 24 & 28 & 513 & 6 & 71 & 73 & 8 & 2 & $?$ & 30 & 29 & 224 & 7 & 43 & 41 & 2 & 8 & 46 & 47 \\
\hline 3 & 25 & 5 & 40 & 42 & 512 & 6 & 45 & 45 & 8 & 1 & 7 & 27 & 25 & 223 & $?$ & 39 & 33 & 2 & 8 & 37 & 32 \\
\hline 3 & 28 & 5 & 48 & 49 & 67 & 6 & 54 & 53 & 8 & 0 & 7 & 52 & 53 & 222 & $?$ & 36 & 43 & 29 & 8 & $6 ?$ & 69 \\
\hline 3 & 29 & 5 & 35 & 31 & 68 & 6 & 56 & 58 & $?$ & 0 & 7 & 33 & 28 & 221 & 7 & 42 & 42 & 210 & 8 & 98 & 99 \\
\hline 3 & 30 & 5 & 23 & 23 & 69 & 6 & 49 & 48 & 7 & 1 & 7 & 38 & 32 & 220 & $?$ & 44 & 49 & 211 & 8 & 69 & 67 \\
\hline 2 & 32 & 5 & 22 & 24 & 610 & 6 & 33 & 35 & 7 & 2 & 7 & 30 & 33 & 219 & $?$ & 52 & 51 & 213 & 8 & 66 & 71 \\
\hline 2 & 31 & 5 & 48 & 47 & 611 & 6 & 42 & 39 & 7 & 3 & 7 & 25 & 21 & 218 & 7 & 36 & 37 & 214 & 8 & 56 & 52 \\
\hline 2 & 28 & 5 & 42 & 44 & 612 & 6 & 61 & 62 & 7 & 5 & 7 & 30 & 25 & 217 & 7 & 56 & 55 & 215 & 8 & 27 & 30 \\
\hline 2 & 27 & 5 & 60 & 58 & 613 & 6 & 55 & 54 & $?$ & 6 & 7 & 39 & 39 & 216 & 7 & 36 & 36 & 217 & 8 & 26 & 25 \\
\hline 2 & 25 & 5 & 43 & 38 & 614 & 6 & 57 & 59 & $?$ & 7 & $?$ & 36 & 35 & 215 & 7 & 36 & 37 & 218 & 8 & 34 & 32 \\
\hline
\end{tabular}


Table 1. Continued.

$\begin{array}{lllll}2 & 19 & 8 & 27 & 34 \\ 2 & 20 & 8 & 25 & 29 \\ 2 & 21 & 8 & 36 & 36 \\ 2 & 23 & 8 & 40 & 42 \\ 3 & 22 & 8 & 44 & 43 \\ 3 & 21 & 8 & 44 & 46 \\ 3 & 20 & 8 & 22 & 15 \\ 3 & 17 & 8 & 28 & 27 \\ 3 & 16 & 8 & 21 & 24 \\ 3 & 15 & 8 & 30 & 34 \\ 3 & 14 & 8 & 50 & 53 \\ 3 & 11 & 8 & 29 & 24 \\ 3 & 10 & 8 & 27 & 32 \\ 3 & 9 & 8 & 29 & 27 \\ 3 & 8 & 8 & 35 & 34 \\ 3 & 7 & 8 & 52 & 51 \\ 3 & 5 & 8 & 67 & 68 \\ 3 & 4 & 8 & 21 & 25 \\ 3 & 3 & 8 & 74 & 76 \\ 3 & 2 & 8 & 21 & 19 \\ 3 & 1 & 8 & 40 & 39 \\ 3 & 0 & 8 & 42 & 42 \\ 4 & 0 & 8 & 69 & 69 \\ 4 & 1 & 8 & 33 & 32 \\ 4 & 2 & 8 & 54 & 52 \\ 4 & 3 & 8 & 35 & 34 \\ 4 & 4 & 8 & 39 & 40 \\ 4 & 5 & 8 & 47 & 49 \\ 4 & 6 & 8 & 47 & 44 \\ 4 & 7 & 8 & 32 & 31 \\ 4 & 8 & 8 & 51 & 49 \\ 4 & 10 & 8 & 29 & 28\end{array}$

$\begin{array}{rllll}4 & 11 & 8 & 35 & 38 \\ 4 & 12 & 8 & 65 & 66 \\ 4 & 15 & 8 & 32 & 31 \\ 4 & 17 & 8 & 22 & 32 \\ 4 & 19 & 8 & 38 & 36 \\ 4 & 20 & 8 & 22 & 26 \\ 5 & 18 & 8 & 23 & 18 \\ 5 & 15 & 8 & 24 & 24 \\ 5 & 14 & 8 & 20 & 12 \\ 5 & 13 & 8 & 29 & 27 \\ 5 & 11 & 8 & 23 & 19 \\ 5 & 10 & 8 & 25 & 23 \\ 5 & 8 & 8 & 35 & 33 \\ 5 & 7 & 8 & 29 & 32 \\ 5 & 5 & 8 & 26 & 22 \\ 5 & 4 & 8 & 32 & 37 \\ 5 & 1 & 8 & 41 & 41 \\ 5 & 0 & 8 & 25 & 28 \\ 6 & 0 & 8 & 26 & 27 \\ 6 & 1 & 8 & 30 & 30 \\ 6 & 2 & 8 & 42 & 38 \\ 6 & 3 & 8 & 60 & 57 \\ 6 & 4 & 8 & 46 & 42 \\ 6 & 5 & 8 & 44 & 45 \\ 6 & 7 & 8 & 37 & 39 \\ 6 & 8 & 8 & 55 & 57 \\ 6 & 11 & 8 & 28 & 20 \\ 6 & 12 & 8 & 41 & 38 \\ 6 & 13 & 8 & 24 & 22 \\ 6 & 14 & 8 & 23 & 16 \\ 6 & 15 & 8 & 23 & 20 \\ 7 & 11 & 8 & 31 & 26\end{array}$

$\begin{array}{rrrll}7 & 8 & 8 & 34 & 34 \\ 7 & 7 & 8 & 30 & 27 \\ 7 & 5 & 8 & 25 & 25 \\ 7 & 4 & 8 & 30 & 27 \\ 7 & 3 & 8 & 42 & 40 \\ 7 & 1 & 8 & 22 & 30 \\ 6 & 1 & 9 & 50 & 47 \\ 5 & 1 & 9 & 30 & 29 \\ 5 & 2 & 9 & 25 & 26 \\ 5 & 3 & 9 & 26 & 27 \\ 5 & 4 & 9 & 61 & 60 \\ 5 & 5 & 9 & 40 & 34 \\ 5 & 7 & 9 & 34 & 33 \\ 5 & 9 & 9 & 41 & 37 \\ 5 & 11 & 9 & 23 & 24 \\ 4 & 14 & 9 & 43 & 47 \\ 4 & 10 & 9 & 57 & 61 \\ 4 & 8 & 9 & 40 & 46 \\ 4 & 6 & 9 & 48 & 48 \\ 4 & 5 & 9 & 32 & 32 \\ 4 & 4 & 9 & 67 & 64 \\ 4 & 0 & 9 & 36 & 35 \\ 3 & 0 & 9 & 32 & 33 \\ 3 & 1 & 9 & 31 & 32 \\ 3 & 2 & 9 & 24 & 23 \\ 3 & 3 & 9 & 54 & 55 \\ 3 & 4 & 9 & 49 & 49 \\ 3 & 5 & 9 & 43 & 48 \\ 3 & 6 & 9 & 41 & 39 \\ 3 & 7 & 9 & 44 & 42 \\ 3 & 8 & 9 & 41 & 42\end{array}$

$\begin{array}{lllll}3 & 9 & 9 & 36 & 32\end{array}$ $\begin{array}{lllll}3 & 10 & 9 & 47 & 44\end{array}$ $\begin{array}{lllll}3 & 11 & 9 & 29 & 23\end{array}$ $\begin{array}{llll}312 & 9 & 30 & 25\end{array}$ $\begin{array}{lllll}3 & 13 & 9 & 43 & 38\end{array}$ $\begin{array}{lllll}2 & 18 & 9 & 39 & 34\end{array}$ $\begin{array}{lllll}2 & 17 & 9 & 22 & 24\end{array}$ $\begin{array}{lllll}2 & 16 & 9 & 35 & 33\end{array}$ $\begin{array}{lllll}2 & 14 & 9 & 20 & 19\end{array}$ $\begin{array}{lllll}2 & 12 & 9 & 35 & 38\end{array}$ $\begin{array}{lllll}2 & 9 & 9 & 46 & 42\end{array}$ $\begin{array}{lllll}2 & 8 & 9 & 61 & 60\end{array}$ $\begin{array}{lllll}2 & 6 & 9 & 48 & 48\end{array}$ $\begin{array}{lllll}2 & 5 & 9 & 44 & 35\end{array}$ $\begin{array}{lllll}2 & 4 & 9 & 41 & 44\end{array}$ $\begin{array}{lllll}2 & 3 & 9 & 68 & 65\end{array}$ $\begin{array}{lllll}2 & 2 & 9 & 23 & 15\end{array}$ $\begin{array}{lllll}2 & 1 & 9 & 30 & 35\end{array}$ $\begin{array}{lllll}1 & 0 & 9 & 24 & 28\end{array}$ $\begin{array}{lllll}1 & 1 & 9 & 50 & 44\end{array}$ $\begin{array}{lllll}1 & 2 & 9 & 37 & 35\end{array}$ $\begin{array}{lllll}1 & 4 & 9 & 20\end{array}$ $16934 \quad 26$ $\begin{array}{lllll}1 & 9 & 9 & 21 & 19\end{array}$ $\begin{array}{lllll}1 & 10 & 9 & 88 & 90\end{array}$ $\begin{array}{lllll}1 & 12 & 9 & 32 & 30\end{array}$ $\begin{array}{lllll}1 & 15 & 9 & 32 & 29\end{array}$ $\begin{array}{lllll}0 & 18 & 9 & 23 & 12\end{array}$ $\begin{array}{llll}0 & 16 & 29 & 31\end{array}$ $\begin{array}{llll}0 & 13 & 9 & 23\end{array}$

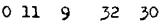

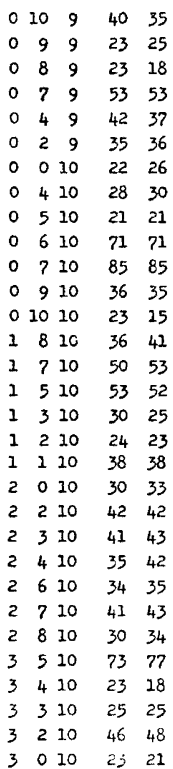

$\begin{array}{llll}0 & 10 \quad 40 \quad 35\end{array}$ $\begin{array}{lll}0 & 49 & 42 \\ 0 & 37\end{array}$ $\begin{array}{lllll}0 & 2 & 9 & 35 & 36\end{array}$ $\begin{array}{lllll}0 & 0 & 10 & 22 & 26\end{array}$ $\begin{array}{llll}0 & 410 \quad 2830\end{array}$ 2121 $0710 \quad 8585$ - $910 \quad 36 \quad 35$ $\begin{array}{llll}0 & 10 \quad 10 \quad 23 \quad 25\end{array}$ $\begin{array}{lllll}1 & 810 & 36 & 41\end{array}$ 17105053 $1510 \quad 53 \quad 52$

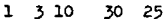
$\begin{array}{lllll}1 & 2 & 10 & 24 & 23\end{array}$ $\begin{array}{lllll}1 & 1 & 10 & 38 & 38\end{array}$ $30 \quad 33$ $\begin{array}{llll}3 & 10 & 41 & 43\end{array}$ $\begin{array}{lll}10 & 35 & 42 \\ 10 & 34 & 35\end{array}$ $\begin{array}{lllll}2 & 7 & 10 & 41 & 43\end{array}$ 2 $810 \quad 30 \quad 34$ $\begin{array}{llll}510 & 73 & 77\end{array}$ $\begin{array}{lllll}3 & 3 & 10 & 25 & 25\end{array}$ $\begin{array}{lllll}3 & 0 & 10 & 25 & 21\end{array}$

Table 2. Fractional atomic coordinates and thermal parameters with estimated standard deviations $\left(\times 10^{5}\right)$ for non-hydrogen atoms. The temperature factor is given by exp $-\left(B_{11} h^{2}+B_{22} k^{2}+B_{33} l^{2}+B_{12} h k+B_{13} h l+B_{23} k l\right)$.

\begin{tabular}{|c|c|c|c|c|c|c|c|c|c|}
\hline Atom & $x$ & $y$ & $z$ & $B_{11}$ & $B_{22}$ & $B_{33}$ & $B_{12}$ & $B_{13}$ & $B_{23}$ \\
\hline $\begin{array}{l}\text { O1 } \\
\text { O1 }\end{array}$ & $\begin{array}{r}48139 \\
19\end{array}$ & $\begin{array}{r}59909 \\
5\end{array}$ & $\begin{array}{r}47104 \\
19\end{array}$ & $\begin{array}{r}1973 \\
30\end{array}$ & $\begin{array}{r}113 \\
2\end{array}$ & $\begin{array}{r}1492 \\
31\end{array}$ & $\begin{array}{r}155 \\
14\end{array}$ & $\begin{array}{r}-303 \\
54\end{array}$ & $\begin{array}{r}3 \\
14\end{array}$ \\
\hline $\mathrm{O} 2$ & $\begin{array}{r}16646 \\
17\end{array}$ & $\begin{array}{r}33312 \\
\\
\quad 5\end{array}$ & $\begin{array}{r}62615 \\
20\end{array}$ & $\begin{array}{r}1491 \\
24\end{array}$ & $\begin{array}{r}156 \\
2\end{array}$ & $\begin{array}{r}1469 \\
30\end{array}$ & $\begin{array}{r}116 \\
14\end{array}$ & $\begin{array}{r}533 \\
53\end{array}$ & $\begin{array}{r}-155 \\
16\end{array}$ \\
\hline O3 & $\begin{array}{r}42813 \\
19\end{array}$ & $\begin{array}{r}28568 \\
5\end{array}$ & $\begin{array}{r}77069 \\
18\end{array}$ & $\begin{array}{r}1830 \\
28\end{array}$ & $\begin{array}{r}173 \\
3\end{array}$ & $\begin{array}{r}879 \\
26\end{array}$ & $\begin{array}{l}87 \\
15\end{array}$ & $\begin{array}{r}-205 \\
50\end{array}$ & $\begin{array}{l}44 \\
14\end{array}$ \\
\hline $\mathrm{N}$ & $\begin{array}{r}24134 \\
20\end{array}$ & $\begin{array}{r}30395 \\
5\end{array}$ & $\begin{array}{r}19616 \\
20\end{array}$ & $\begin{array}{r}1352 \\
26\end{array}$ & $\begin{array}{r}109 \\
2\end{array}$ & $\begin{array}{r}910 \\
28\end{array}$ & $\begin{array}{r}-3 \\
14\end{array}$ & $\begin{array}{r}-104 \\
50\end{array}$ & $\begin{array}{l}39 \\
15\end{array}$ \\
\hline $\mathrm{C} 1$ & $\begin{array}{r}54146 \\
21\end{array}$ & $\begin{array}{r}40561 \\
6\end{array}$ & $\begin{array}{r}35382 \\
24\end{array}$ & $\begin{array}{r}905 \\
26\end{array}$ & $\begin{array}{r}104 \\
2\end{array}$ & $\begin{array}{r}1116 \\
34\end{array}$ & $\begin{array}{r}-63 \\
14\end{array}$ & $\begin{array}{r}119 \\
54\end{array}$ & $\begin{array}{l}60 \\
16\end{array}$ \\
\hline C2 & $\begin{array}{r}59675 \\
23\end{array}$ & $\begin{array}{r}43336 \\
6\end{array}$ & $\begin{array}{r}56092 \\
25\end{array}$ & $\begin{array}{r}1145 \\
29\end{array}$ & $\begin{array}{r}123 \\
3\end{array}$ & $\begin{array}{r}1044 \\
33\end{array}$ & $\begin{array}{r}-19 \\
16\end{array}$ & $\begin{array}{r}-299 \\
58\end{array}$ & $\begin{array}{r}103 \\
17\end{array}$ \\
\hline
\end{tabular}

Acta Chem. Scand. 26 (1972) No. 10 
Table 2. Continued.

\begin{tabular}{rrrrrrrrrr} 
C3 & 57417 & 49774 & 59840 & 1214 & 123 & 916 & -73 & -273 & -25 \\
& 24 & 6 & 25 & 29 & 3 & 33 & 16 & 56 & 16 \\
$\mathrm{C} 4$ & 49410 & 53547 & 42897 & 930 & 104 & 1191 & 6 & 126 & 41 \\
& 21 & 6 & 25 & 26 & 3 & 34 & 14 & 57 & 17 \\
$\mathrm{C} 5$ & 43388 & 50888 & 22293 & 1154 & 126 & 1182 & 3 & -448 & 147 \\
& 24 & 7 & 26 & 30 & 3 & 36 & 16 & 61 & 17 \\
$\mathrm{C} 6$ & 45900 & 44421 & 18657 & 1181 & 131 & 1024 & -101 & -301 & 19 \\
& 23 & 7 & 25 & 29 & 3 & 33 & 16 & 55 & 17 \\
$\mathrm{C} 7$ & 57507 & 33558 & 31222 & 1127 & 109 & 1322 & 64 & 298 & -18 \\
& 23 & 7 & 26 & 28 & 3 & 35 & 15 & 60 & 17 \\
$\mathrm{C} 8$ & 40003 & 29337 & 36562 & 1164 & 87 & 838 & 48 & -44 & -20 \\
& 21 & 6 & 23 & 28 & 2 & 29 & 14 & 54 & 15 \\
$\mathrm{C} 9$ & 32403 & 30518 & 60854 & 1339 & 81 & 904 & -119 & 282 & -69 \\
& 24 & 6 & 24 & 29 & 2 & 31 & 15 & 53 & 15 \\
\hline & & & & & & & & &
\end{tabular}

Table 3. Fractional atomic coordinates $\left(\times 10^{4}\right)$ and isotopic thermal parameters $\left(\AA^{2}\right)$ with estimated standard deviations for hydrogen atoms.

\begin{tabular}{|c|c|c|c|c|}
\hline Atom & $x$ & $y$ & $z$ & $B$ \\
\hline $\mathrm{H} 2$ & $\begin{array}{r}6559 \\
29\end{array}$ & $\begin{array}{r}4068 \\
10\end{array}$ & $\begin{array}{r}6705 \\
39\end{array}$ & $\begin{array}{l}1.88 \\
0.38\end{array}$ \\
\hline H3 & $\begin{array}{r}6100 \\
30\end{array}$ & $\begin{array}{r}5177 \\
10\end{array}$ & $\begin{array}{r}7350 \\
38\end{array}$ & $\begin{array}{l}2.05 \\
0.40\end{array}$ \\
\hline H5 & $\begin{array}{r}3758 \\
28\end{array}$ & $\begin{array}{r}5355 \\
9\end{array}$ & $\begin{array}{r}1090 \\
37\end{array}$ & $\begin{array}{l}1.96 \\
0.36\end{array}$ \\
\hline $\mathrm{H} 6$ & $\begin{array}{r}4211 \\
34\end{array}$ & $\begin{array}{r}4255 \\
10\end{array}$ & $\begin{array}{r}316 \\
38\end{array}$ & $\begin{array}{l}2.60 \\
0.43\end{array}$ \\
\hline $\mathrm{H} 7 \mathrm{I}$ & $\begin{array}{r}6815 \\
28\end{array}$ & $\begin{array}{r}3207 \\
8\end{array}$ & $\begin{array}{r}4028 \\
38\end{array}$ & $\begin{array}{l}2.01 \\
0.37\end{array}$ \\
\hline $\mathrm{H} 72$ & $\begin{array}{r}6143 \\
32\end{array}$ & $\begin{array}{r}3279 \\
11\end{array}$ & $\begin{array}{r}1442 \\
45\end{array}$ & $\begin{array}{l}3.21 \\
0.48\end{array}$ \\
\hline H8 & $\begin{array}{r}4392 \\
23\end{array}$ & $\begin{array}{r}2501 \\
7\end{array}$ & $\begin{array}{r}3462 \\
27\end{array}$ & $\begin{array}{l}0.59 \\
0.26\end{array}$ \\
\hline HOl & $\begin{array}{r}4423 \\
44\end{array}$ & $\begin{array}{r}6154 \\
12\end{array}$ & $\begin{array}{r}3819 \\
55\end{array}$ & $\begin{array}{l}3.71 \\
0.66\end{array}$ \\
\hline HN 1 & $\begin{array}{r}1976 \\
28\end{array}$ & $\begin{array}{r}3415 \\
10\end{array}$ & $\begin{array}{r}1934 \\
39\end{array}$ & $\begin{array}{l}1.33 \\
0.39\end{array}$ \\
\hline HN2 & $\begin{array}{r}1445 \\
28\end{array}$ & $\begin{array}{r}2780 \\
9\end{array}$ & $\begin{array}{r}2227 \\
37\end{array}$ & $\begin{array}{l}1.94 \\
0.38\end{array}$ \\
\hline HN3 & $\begin{array}{r}2981 \\
31 \\
\end{array}$ & $\begin{array}{r}3002 \\
10 \\
\end{array}$ & $\begin{array}{r}542 \\
43 \\
\end{array}$ & $\begin{array}{l}2.89 \\
0.44 \\
\end{array}$ \\
\hline
\end{tabular}

Acta Chem. Scand. 26 (1972) No. 10 
rather than the molecule as a whole. The phenol part has the axis of the largest oscillation amplitude nearly parallel to the $\mathrm{C} 7-\mathrm{C} 1-\mathrm{C} 4-\mathrm{O} 1$ direction whereas the corresponding axis of the alanine moiety is roughly perpendicular to the $\mathrm{Cl}$ - C7-C8 plane. The bond lengths were corrected for librational effects according to this model.

Standard deviations were calculated from the correlation matrix ignoring the standard deviations in cell parameters.

Table 4. R.m.s. amplitudes of vibration $\left(\bar{u}^{2}\right)^{\frac{1}{2}}(\AA)$ and $B$-values $\left(\AA^{2}\right)$ along the principal axes of vibration given by the components of a unit vector $e$ in fractional coordinates $\left(\times 10^{3}\right)$.

\begin{tabular}{|c|c|c|c|c|c|}
\hline Atom & $\left(\bar{u}^{2}\right)^{\frac{1}{2}}$ & $B$ & $e_{x}$ & $e_{y}$ & $e_{z}$ \\
\hline 01 & $\begin{array}{l}.223 \\
.160 \\
.154\end{array}$ & $\begin{array}{l}3.91 \\
2.03 \\
1.88\end{array}$ & $\begin{array}{r}215 \\
0 \\
040\end{array}$ & $\begin{array}{r}51 \\
77 \\
-131\end{array}$ & $\begin{array}{r}-428 \\
141 \\
72\end{array}$ \\
\hline $\mathrm{O} 2$ & $\begin{array}{l}.200 \\
.190 \\
.143\end{array}$ & $\begin{array}{l}3.16 \\
2.85 \\
1.61\end{array}$ & $\begin{array}{r}158 \\
89 \\
-57\end{array}$ & $\begin{array}{r}121 \\
-132 \\
56\end{array}$ & $\begin{array}{r}19 \\
104 \\
119\end{array}$ \\
\hline O3 & $\begin{array}{l}.214 \\
.194 \\
.122\end{array}$ & $\begin{array}{l}3.62 \\
2.98 \\
1.17\end{array}$ & $\begin{array}{r}194 \\
80 \\
-10\end{array}$ & $\begin{array}{r}90 \\
176 \\
-81\end{array}$ & $\begin{array}{r}-92 \\
18 \\
121\end{array}$ \\
\hline $\mathbf{N}$ & $\begin{array}{l}.181 \\
.157 \\
.124\end{array}$ & $\begin{array}{l}2.59 \\
1.96 \\
1.22\end{array}$ & $\begin{array}{r}81 \\
5 \\
8\end{array}$ & $\begin{array}{r}4 \\
156 \\
-16\end{array}$ & $\begin{array}{r}12 \\
20 \\
123\end{array}$ \\
\hline $\mathrm{Cl}$ & $\begin{array}{l}.159 \\
.148 \\
.132\end{array}$ & $\begin{array}{l}2.00 \\
1.72 \\
1.39\end{array}$ & $\begin{array}{r}-82 \\
108 \\
59\end{array}$ & $\begin{array}{r}134 \\
50 \\
55\end{array}$ & $\begin{array}{r}25 \\
87 \\
-105\end{array}$ \\
\hline $\mathrm{C} 2$ & $\begin{array}{l}.173 \\
.164 \\
.128\end{array}$ & $\begin{array}{l}2.37 \\
1.13 \\
2.29\end{array}$ & $\begin{array}{r}-114 \\
118 \\
30\end{array}$ & $\begin{array}{r}116 \\
115 \\
-32\end{array}$ & $\begin{array}{r}60 \\
1 \\
120\end{array}$ \\
\hline C3 & $\begin{array}{l}.178 \\
.162 \\
.123\end{array}$ & $\begin{array}{l}2.49 \\
2.07 \\
1.19\end{array}$ & $\begin{array}{r}-147 \\
85 \\
26\end{array}$ & $\begin{array}{r}98 \\
134 \\
13\end{array}$ & $\begin{array}{r}21 \\
-33 \\
120\end{array}$ \\
\hline $\mathrm{C4}$ & $\begin{array}{l}.156 \\
.151 \\
.140\end{array}$ & $\begin{array}{l}1.91 \\
1.79 \\
1.55\end{array}$ & $\begin{array}{r}65 \\
125 \\
52\end{array}$ & $\begin{array}{r}126 \\
-79 \\
38\end{array}$ & $\begin{array}{r}66 \\
28 \\
-124\end{array}$ \\
\hline C5 & $\begin{array}{l}.178 \\
.168 \\
.129\end{array}$ & $\begin{array}{l}2.51 \\
2.23 \\
1.32\end{array}$ & $\begin{array}{r}-101 \\
124 \\
46\end{array}$ & $\begin{array}{r}118 \\
112 \\
-44\end{array}$ & $\begin{array}{r}88 \\
-07 \\
112\end{array}$ \\
\hline $\mathrm{C} 6$ & $\begin{array}{l}.182 \\
.160 \\
.130\end{array}$ & $\begin{array}{l}2.62 \\
2.03 \\
1.33\end{array}$ & $\begin{array}{r}-125 \\
109 \\
34\end{array}$ & $\begin{array}{r}130 \\
113 \\
4\end{array}$ & $\begin{array}{r}29 \\
-34 \\
125\end{array}$ \\
\hline
\end{tabular}

Acta Chem. Scand. 26 (1972) No. 10 
Table 4. Continued.

\begin{tabular}{rrrrrr} 
& & & & \\
$\mathrm{C} 7$ & .172 & 2.33 & 148 & 63 & 61 \\
& .157 & 1.94 & 23 & -132 & 81 \\
& .143 & 1.62 & -70 & 57 & 111 \\
$\mathrm{C} 8$ & .169 & 2.25 & 166 & 34 & -7 \\
& .139 & 1.53 & 28 & -135 & 15 \\
& .120 & 1.13 & 2 & 13 & 119 \\
& & & & & \\
$\mathrm{C} 9$ & .185 & 2.71 & 175 & -53 & 33 \\
& .133 & 1.39 & 44 & 112 & -56 \\
& .120 & 1.13 & -4 & 54 & 107 \\
\hline
\end{tabular}

\section{DISCUSSION}

A drawing of the molecule is shown in Fig. 1 in which the numbering of the atoms is indicated. The bond lengths (corrected) and valence angles are given in this figure, as well as in Table 5 in which the estimated standard deviations are also listed. The results presented are confirmed by a neutron diffraction study of the compound. ${ }^{10}$

In the alanine moiety the usual zwitterionic nature of a free amino acid is indicated both by the nearly equal carbon-oxygen bond length in the carboxyl group and the tetrahedral arrangement of hydrogen atoms around the nitrogen atom. The conformation about the $\mathrm{N}-\mathrm{C} 8$ bond is staggered.

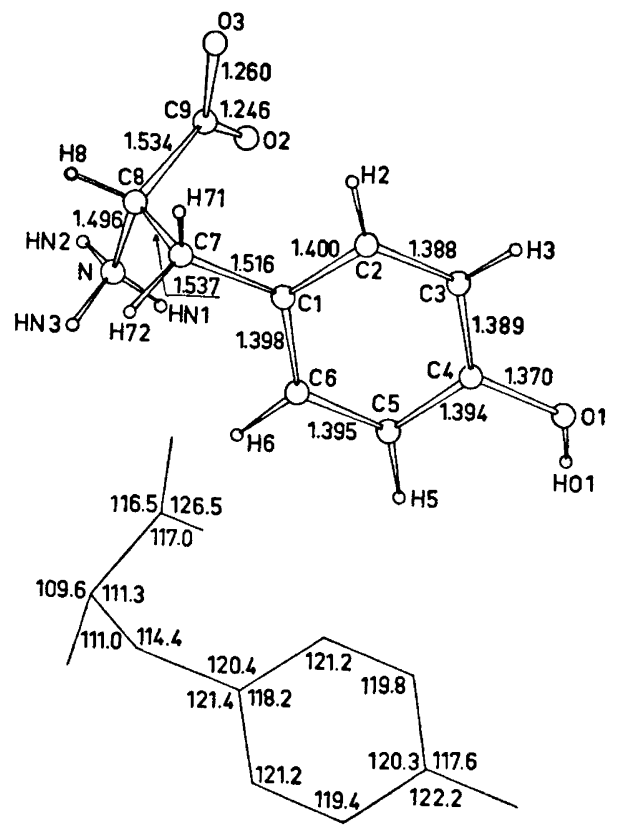

Fig. 1. Bond lengths $(\AA)$ corrected for thermal vibration effects and angles $\left({ }^{\circ}\right)$ in $\mathrm{L}_{4}$-tyrosine.

Acta Chem. Scand. 26 (1972) No. 10 
Table 5. Bond lengths $(\AA)$ and bond angles $\left({ }^{\circ}\right)$. Estimated standard deviations (in parentheses) apply to the least significant digits.

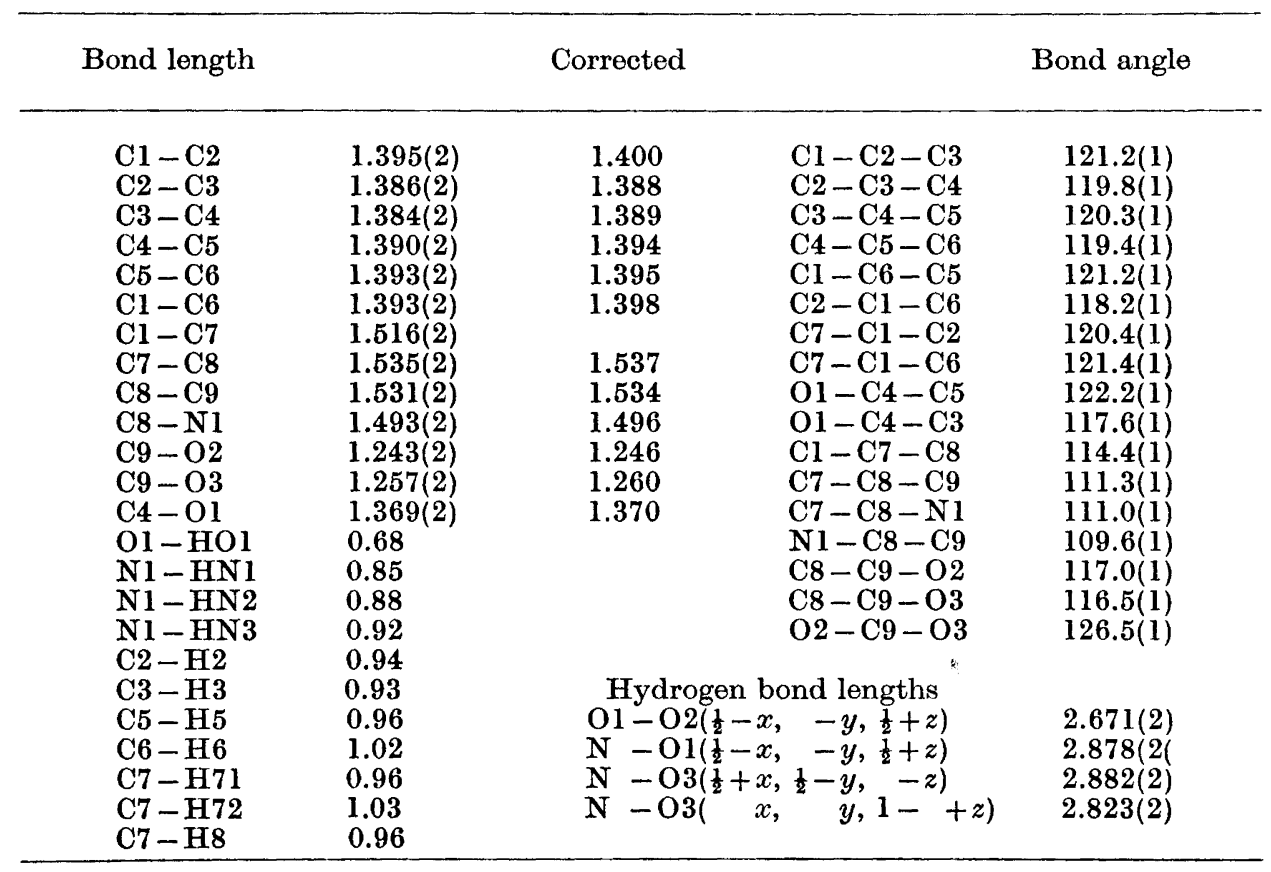

This appears to be a general feature in amino acids even in the solid state where the energy of formation of hydrogen bonds is supposed to be greater than the energy barriers of rotation of the $\mathrm{C}-\mathrm{NH}_{3}$ bond $(2-3 \mathrm{kcal})$. The preferred orientation of this group seems to be an important factor in the crystallization process in such a way that the requirements for a staggered conformation and the tetrahedral distribution of hydrogen bonds about the ammonium group can both be met with in the crystal. ${ }^{11}$ This is an interesting point in the discussion of the degree of correspondence between the conformation of a molecule in the crystal as compared to that present in solution and in the gas phase. The $\mathrm{C}-\mathrm{NH}_{3}{ }^{+}$bond length (1.496 $\AA$ ) is within the range normally reported for such bonds. ${ }^{12-14}$ The C8 - C9 bond length (1.534 $\AA$ ) also closely agrees with the value found in other $\alpha$-amino acids but is longer than the normal $\mathrm{C}\left(s p^{3}\right)-\mathrm{C}\left(s p^{2}\right)$ single bond..$^{15}$

The geometry of the acid group is nearly identical to what was found in dihydroxyphenylalanine, ${ }^{12}$ the carbon-oxygen bonds $(1.246 \AA$ and $1.260 \AA)$ are not quite equal, the oxygen atom of the longer bond being involved in two hydrogen bonds whereas the other has only one such contact.

The nitrogen atom is situated $0.35 \AA$ from the plane of the carboxyl group and the torsional angle $\mathrm{N}-\mathrm{C} 8-\mathrm{C} 9-\mathrm{O} 2$ is $14^{\circ}$, which is close to the mean value for this angle..$^{15}$

Acta Chem, Scand. 26 (1972) No. 10 
The conformation about the $\mathrm{C} 7-\mathrm{C} 8$ bond is staggered, the $\mathrm{Cl}-\mathrm{C} 7-\mathrm{C} 8-\mathrm{C} 9$ angle being $53.1^{\circ}$ and the $\mathrm{C} 1-\mathrm{C} 7-\mathrm{C} 8-\mathrm{N} 290.7^{\circ}$. Both the ammonium and the carboxyl groups are thus in gauche positions relative to the phenol moiety. The angle between the benzene ring plane and the one defined by the $\mathrm{Cl}$ $\mathrm{C} 7-\mathrm{C} 8$ atoms is $85.9^{\circ}$.

The $\mathrm{Cl}-\mathrm{C} 7$ bond length is $1.516 \AA$ as compared to $1.511 \AA$ in L-tyrosine$O$-sulfate dihydrate ${ }^{14}$ and $1.512 \AA$ in $\mathrm{L}_{\text {-DOPA. }}{ }^{12}$

The six atoms of the benzene ring are coplanar. Their deviations from a least-squares plane are given in Table 6 .

Table 6. Deviations ( $\AA$ ) from a least-squares plane through the benzene ring atoms.

$\begin{array}{lrrrrrrr}\text { C1 } & 0.007 & \text { C3 } & -0.001 & \text { C5 } & -0.007 & \text { O1 } & 0.063 \\ \text { C2 } & -0.007 & \text { C4 } & 0.008 & \text { C6 } & 0.000 & \text { C7 } & 0.060\end{array}$

The oxygen and carbon atoms bonded directly to the ring atoms are situated significantly out of this plane. The hydrogen atom $\mathrm{HOl}$ is close to the benzene ring plane.

The $\mathrm{C} 4-\mathrm{Ol}$ bond length is found to be $1.370 \AA$ in accordance with what is usually observed in phenols and catechols but somewhat shorter than what might be expected from the covalent radii of $\mathrm{C}\left(s p^{2}\right)$ and $\mathrm{O}$; a certain degree of delocalization of the electrons from the oxygen atom may account for this effect as well as for the tendency for the phenolic hydrogen atom to lie in the plane of the benzene ting. The external bond angles at the $\mathrm{C} 4$ atom are deformed relative to a symmetric arrangement in the same way as found in $\mathrm{L}_{\mathrm{L}}-\mathrm{DOPA}{ }^{\mathbf{1 2}}$ and similar structures.

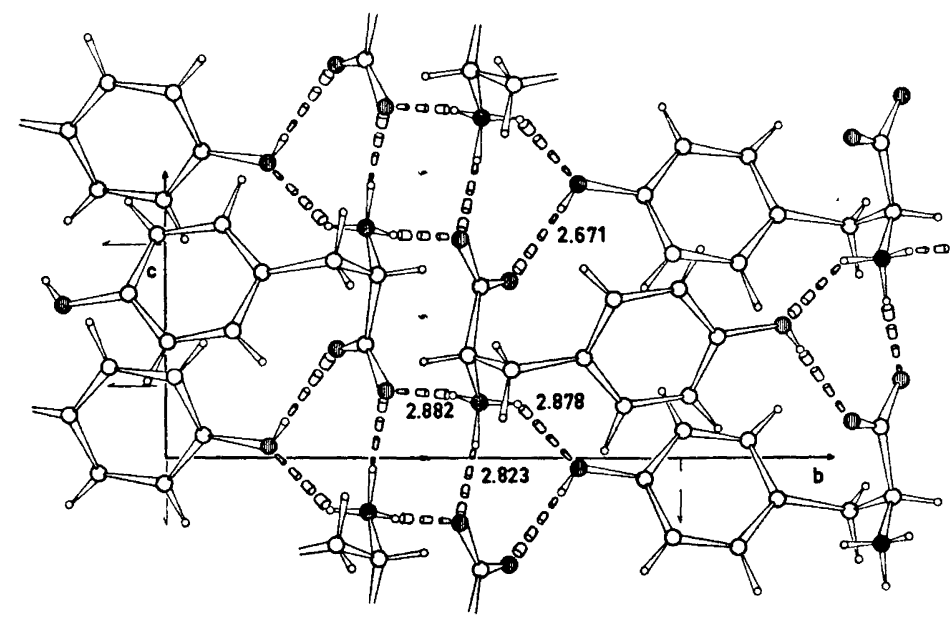

Fig. 2. The crystal structure as seen down the $a$-axis. The hydrogen bond lengths $(\AA)$ are indicated. 


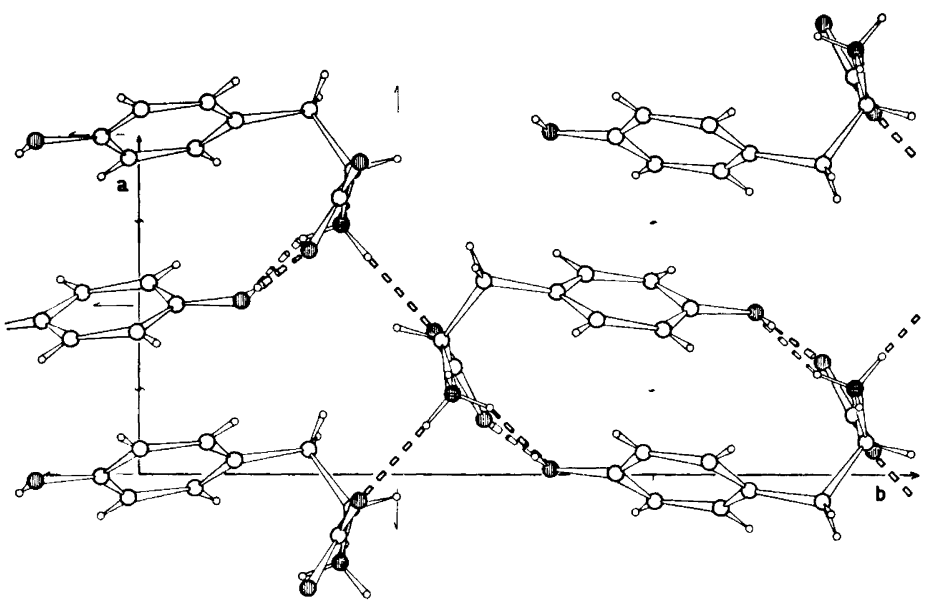

Fig. 3. The crystal structure as seen along the $c$-axis.

The mean carbon-carbon bond length in the benzene ring is $1.394 \AA$ with a maximum deviation of $0.006 \AA$.

A crystal structure is characterized by a three-dimensional hydrogen bond network as visualized in Figs. 2 and 3. The nitrogen atom is hydrogen donor in three hydrogen bonds, two to carboxyl oxygen atoms of two different molecules (2.882 and $2.823 \AA$ ) and one to a phenolic oxygen atom of a third $(2.878 \AA)$. The phenolic hydrogen atom participate in a hydrogen bond to a carboxylic oxygen atom of a neighbouring molecule (2.671 $\AA$ ). Each L-tyrosine molecule is hydrogen bonded to six neighbouring molecules.

Acknowledgement. The authors express their gratitude to cand. real. Per Groth for his advice in connection with the practical applications of direct methods.

\section{REFERENCES}

1. Mostad, A., Nissen, H. M. and Rømming, C. Acta Chem. Scand. 25 (1971) 1145.

2. Mostad, A., Nissen, H. M. and Rømming, C. Tetrahedron Letters 24 (1971) 2131.

3. Boggs, R. and Donohue, J. Acta Cryst. B 27 (1971) 247.

4. Dahl, T., Gram, F., Groth, P., Klewe, B. and Rømming, C. Acta Chem. Scand. 24 (1970) 2232.

5. Hanson, H. P., Herman, F., Lea, J. D. and Skillman, S. Acta Cryst. 17 (1964) 1040.

6. Stewart, R. F., Davidson, E. R. and Simpson, W. T. J. Chem. Phys. 42 (1965) 3175.

7. Karle, J. and Karle, I. L. Acta Cryst. 21 (1966) 849.

8. Karle, J. and Hauptman, M. Acta Cryst. 9 (1956) 635.

9. Stanley, E. Acta Cryst. 17 (1964) 1028.

10. Lehman, M. and Frey, M. Private communication.

11. Kanters, J. A., Kroon, J., Beurskens, P. T. and Vliegenhart, J. A. Acta Cryst. 21 (1966) 990.

12. Mostad, A., Ottersen, T. and Rømming, C. Acta Chem. Scand. 25 (1971) 3549.

13. Bye, E., Mostad, A. and Rømming, C. Acta Chem. Scand. 25 (1971) 364.

14. Fries, D. C. and Sundaralingman, M. Acta Cryst. B 27 (1971) 401.

15. Sundaralingam, M. and Putkey, E. F. Acta Cryst. B 26 (1970) 790.

Received May 2, 1972. 